\title{
Kronian Magnetospheric Reconnection Statistics Across Cassini's Lifetime
}

\author{
T. M. Garton ${ }^{1, *}$, C. M. Jackman ${ }^{2}$, A. W. Smith ${ }^{3}$ \\ ${ }^{1}$ Space Environment Physics Group, Department of Physics and Astronomy, University of Southampton, \\ ${ }^{2}$ School of Cosmic Physics, DIAS Dunsink Observatory, Dublin Institute for Advanced Studies, Dublin 15,

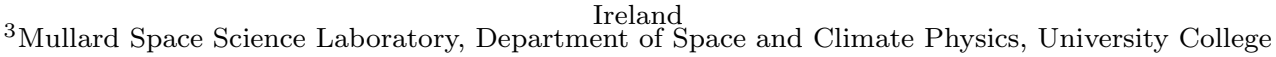 \\ London, London, England
}

\section{Key Points:}

- Machine learning classifications in previously unobserved environments can be validated through iterative runs.

- The Cassini data do not reveal a quasi-steady magnetotail reconnection x-line inside of $<70 \mathrm{R}_{S}$.

- Cassini observations indicate a mass loss rate between $1.50-44.87 \mathrm{~kg} \mathrm{~s}^{-1}$ due to magnetotail plasmoid release.

Corresponding author: Tadhg Garton, t.m.garton@soton.ac.uk

This article has been accepted for publication and ${ }^{-1}$ undergone full peer review but has not been through the copyediting, typesetting, pagination and proofreading process, which may lead to differences between this version and the Version of Record. Please cite this article as doi: 10.1029/2021JA029361.

This article is protected by copyright. All rights reserved. 


\begin{abstract}
Magnetic reconnection is a fundamental physical process in planetary magnetospheres, in which plasma can be exchanged between the solar wind and a planetary magnetosphere, and material can be disconnected and ultimately lost from a magnetosphere. Magnetic reconnection in a planetary magnetotail can result in the release of plasmoids downtail and dipolarizations planetward of an $\mathrm{x}$-line. The signatures of these products include characteristic deflections in the north-south component of the magnetic field which can be detected by in-situ spacecraft. These signatures have been identified by eye, semi-automated algorithms, and recently machine learning methods. Here, we apply statistical analysis to the most thorough catalogue of Kronian magnetospheric reconnection signatures created through machine learning methods to improve understanding of magnetospheric evolution. This research concludes that no quasi-steady position of the magnetotail x-line exists within $70 \mathrm{R}_{S}$. This research introduces prediction equations to estimate the distribution of duration of plasmoid passage over the spacecraft $\left(N=300 \Delta t^{-1.3}\right.$, bin width $\left.=1 \mathrm{~min}\right)$ and north-south field deflection $\left(N=52 \Delta B_{\theta}^{-2.1}\right.$, bin width $\left.=0.25 \mathrm{nT}\right)$ expected to be identified by an orbiting spacecraft across a year of observations. Furthermore, this research finds a local time asymmetry for reconnection identifications, with a preference for dusk-side over dawn-side. This may indicate a preference for Vasyliunas style reconnection over Dungey style for Saturn. Finally, through these distributions, the reconnection rate of Saturn's magnetotail can be estimated as 3.22 reconnection events per day, with a resulting maximum mass loss from plasmoids of $44.87 \mathrm{~kg} \mathrm{~s}^{-1}$ on average, which is comparable with the magnetospheric mass loading from Enceladus $\left(8-250 \mathrm{~kg} \mathrm{~s}^{-1}\right)$.
\end{abstract}

\title{
1 Introduction
}

Magnetic reconnection is the process whereby a magnetic field enters a state of stress or strain and restructures itself into a lower energy state (Hesse \& Cassak, 2020). This often occurs through the explosive snapping and reforging of magnetic field lines, where mass can be released in the form of plasmoids tailward of the reconnection site. For planets with well-developed magnetospheres, reconnection between the interplanetary magnetic field and planetary magnetic field on the day-side of a magnetosphere can result in the transfer of energy, mass and momentum (Milan et al., 2007; McAndrews et al., 2008). Similarly, on the night-side, open magnetic field lines become stretched into a extended planetary magnetotail which facilitates reconnection to again form closed field lines (Dungey, 1961; Dungey, 1965). This cyclic transition between open and closed field configurations allows the transfer of mass and energy, both in and out, of the planetary magnetosphere, as well as alters the ratio of open-closed magnetic flux to the magnetosphere. Alternatively, reconnection can occur for rapidly rotating magnetized planets, such as Saturn, which involves no variation in overall magnetic flux. For these planets, rapid rotation rates and significant internal mass sources result in the operation of the Vasyliunas cycle where mass is lost down the magnetotail through the reconnection of centrifugally stretched, mass loaded field lines (Vasyliunas, 1983).

Figure 1 illustrates a model of night-side magnetospheric reconnection occurring within the planetary current sheet in a Kronocentric Solar Magnetospheric (KSM) Saturncentered system. This coordinate system is $\mathrm{x}$ axis positive towards the Sun, the $\mathrm{z}$ axis is positive northwards such that the x-z plane contains Saturn's magnetic dipole axis, and the positive y axis points toward dusk. Direct encounters with the reconnection site are extremely rare: at Saturn there has been one reported observation of the ion diffusion region (Arridge et al., 2015). The vast majority of reconnection-related knowledge has been derived from in situ encounters with the products of reconnection: plasmoids, travelling compression regions and dipolarizations, all of which leave characteristic signatures in field and particle data. On the planet-side of the reconnection site reconnection can be identified indirectly by spacecraft through dipolarizations, when the north-south magnetic field undergoes a negative to positive deflection caused by a contracting of reconnected magnetic field back towards the planet (Bunce et al., 2005; Russell et al., 2008; Jackman et al., 2013, 2015; Yao et al., 2017; Smith et al., 2018a, 2018b). On the tail-side, reconnection can be remotely identified through plasmoids (Hones, 1977; Richardson et al., 1987; Jackman et al., 2007; Hill et al., 2008; Jackman et al., 2011a) or travelling compression regions (TCRs ; Slavin et al. (1984)), when the north-south magnetic field undergoes a 
Figure 1. Model of magnetic reconnection in a planetary current sheet. From this form of reconnection, various structures are created: dipolarizations, plasmoids and TCRs which are detectable by in-situ spacecraft through their unique magnetic deflections (adapted from Garton et al. (2021)). These figures are described in a KSM coordinate system.

severe or moderate positive to negative deflection. This is caused by either a plasmoid, enclosed bubble of magnetized plasma, or TCR, compressed bulge in the current sheet, travelling over the observing spacecraft. All of these signatures imply the bulk motion of plasma and hence, the transport of both energy and mass about the magnetosphere. Analysis on the transport of mass focuses on solving the mass budget for Saturn's magnetosphere. Saturn receives a mass loading of plasma from Enceladus of $8-250 \mathrm{~kg} \mathrm{~s}^{-1}$ (Jurac \& Richardson, 2005; Pontius Jr. \& Hill, 2009; Chen et al., 2010; Fleshman et al., 2010). The MHD simulation of Zieger et al. (2010) estimated that plasmoids account for $8 \%$ of the total mass lost down-tail. Bagenal and Delamere (2011) estimated that 200 plasmoids per day would be required to remove $100 \mathrm{~kg} \mathrm{~s}^{-1}$ by assuming a plasmoid of volume $\left(10 \mathrm{R}_{S}\right)^{3}$ with a density of $0.01 \mathrm{~cm}^{-3}$ of 18 atomic mass units (amu) ions. Jackman et al. (2014) instead estimated a distribution of 3.6-196 tail-width plasmoids per day to remove $100 \mathrm{~kg} \mathrm{~s}^{-1}$ with a density of $0.1 \mathrm{~cm}^{-3}$ of $16 \mathrm{amu}$ ions (Thomsen et al., 2014), assuming plasmoids occupy dimensions of: height $=4 R_{S}$, length $=4.28 R_{S}$, and width $=90 R_{S}$. The large-scale Dungey cycle of opening of flux via dayside reconnection, followed by closure of flux on the nightside and loss of material downtail is not the only method by which mass can be added to or removed from the magnetosphere. (Neupane et al., 2019) empirically derived an outflow of mass at $55 \mathrm{~kg} \mathrm{~s}-1$ through observation of plasma flow patterns. Several authors have considered the consequences of a more viscous-like interaction with the solar wind, which can lead to features such as Kelvin-Helmholtz instabilities near the magnetospheric boundaries (e.g. (Masters, 2018)). Such interaction facilities more magnetic sheer stress at the boundaries and can create a local time asymmetry in plasma flow and perhaps in associated mass loss (e.g. (B. Burkholder et al., 2017)).

Numerous studies have been performed on magnetospheric phenomena to understand the global impact of reconnection, ranging from changing plasma flow patterns, to dynamic auroral emissions in UV and radio wavelengths. Plasma flow patterns have been analyzed to understand the difference that reconnection makes to the pattern of sub-corotational, azmiuthally-directed flow, and have been used to search for evidence of an x-line, where one might expect oppositely directed flows on either side (McAndrews et al., 2009; Imber et al., 2011; Thomsen et al., 2014; Neupane et al., 2019). It is noteworthy that the search for Saturn's planetary x-line has not yet been conclusive on a specific location. In addition to in-situ plasma and energetic particle investigations, remote sensing of auroral emissions in multiple wavelengths can give global context to the impact of reconnection. On the radio side, Saturn Kilometric Radiation (SKR) has been observed to both intensify and extend to lower frequencies in response to solar wind compression and magnetotail reconnection events (Jackman et al., 2009; Reed et al., 2018). Furthermore, Saturn's UV aurora, formed at the boundary between open and closed field lines, can act as a diagnostic of the flux content of the magnetosphere, with the oval latitude changing in direct response to opening and closing of flux through reconnection (Badman et al., 2016; Bader et al., 2019; Jasinski et al., 2019). To date, these phenomena have been investigated primarily through case study observations or semi-automatically made catalogues (Bunce et al., 2005; Jackman et al., 2007; Jackman et al., 2008; Hill et al., 2008; Smith et al., 2016). However, the power of machine learning (ML) is that it enables us to explore these phenomena over wider timescales, with larger catalogues of events, and by reducing the bias associated with by-eye selection of events.

The implementation of ML to space physics is a relatively new concept, but a promising one for the improvements to identification, classification and forecasting in the field (Azari et al., 2020). ML's strength is three-fold: its robust unbiased results, its rapid turn around from input to output, and it does not assume or require a specific analytical form or magnetic signature (Smith et al., 2017; Huang et al., 2018). ML operates through the training of a base architecture with a prepared dataset. The prepared dataset 
Figure 2. Trajectory of Cassini spacecraft from Saturn Orbit Insertion (July 2004) to mis-

sion end (September 2017) described in terms of its variation in radius, latitude, and local time

for each orbit. Red points indicate the location of the spacecraft at apoapsis, and blue points

indicate location at periapsis.

will be composed of a set of input properties and a corresponding output classification or property. The weights and biases of the base structure are gradually tuned until for the given training inputs it returns outputs with a reasonable accuracy to the expected results. This can result in over-training, where the ML model has become highly specialized to identify the training inputs with incredible accuracy, but has not learned the true underlying structure that the creator wishes to identify. To curtail this problem, the ML models are compared against new, already classified datasets in a test/validation environment. The accuracy achieved in this test environment represents the model's true ability to classify input datasets to correct outputs (Lapedes \& Farber, 1987; Jabbar \& Khan, 2015; Ying, 2019). The result is a model that is efficient and accurate at identifying correct outputs for given input data. Furthermore this model is consistent: for the given input the model will always return the same output. This is contrasted with human observers, who are highly subject to unquantifiable mis-classifications, uncertainty, and bias. For a single event, two human observers may class it differently, or even the same human observer will classify datasets differently on different passes through a dataset, including being biased by the order in which data are examined. ML models are also extremely rapid with their classifications, completing potentially millions of classifications per second, far outperforming a human classifier, allowing scientific exploitation of a greater volume of data.

Garton et al. (2021) (G21) applied neural network ML methods to Cassini magnetometer data, utilizing the Smith et al. (2016) (S16) catalogue as a training set, to create a Kronian magnetospheric reconnection classifier. The S16 catalogue was created from a semi-automated classification algorithm to identify magnetotail signals of reconnection in Cassini magnetometer data through quadratic fitting and parametric thresholding. The G21 model then classified the entirety of Cassini's near-Saturn lifetime (2004-2017; see Figure 2) rendering the most complete database of magnetic field deflections. This catalogue contains start and end times of identified reconnection events, the spatial location of detection, as well as parametric information, such as the magnitude of the deflection of the north-south field component $\left(\Delta B_{\theta}\right)$ and signal to noise ratio of the observed event. Here, we apply statistical analysis to G21 to further understanding of Saturn's magnetic topology and its seasonal evolution, as well as introduce statistical predictability to magnetospheric reconnection events. Section 2 describes catalogue validation and data pruning applied to the G21 catalogue to ensure statistical analysis is only performed on validated magnetotail events. Section 3 shows a statistical analysis across temporal, spatial, and parametric properties of validated reconnection identifications. Finally, Section 4 investigates the results of this statistical analysis and discusses the improvements to understanding of magnetospheric dynamics.

\section{Catalogue Stability and Reliability}

Machine learning is a powerful tool that can be used in improving the identification and forecasting of events in the scientific sector. However, this new method introduces unforeseen errors and complications, most notably the difficulty on interpreting machine learning architectures. This difficulty of interpretation is not due to a 'black-box'-like nature, but due to the sheer complexity and size of the architectures. While not uninterpretable, an investigation of the architecture is extremely time consuming and hence undermines the principle strength of ML methods, to save time on identification. It is important to distinguish that while these algorithms are poor at interpreting the why of events, i.e. searching for deeper meaning behind datasets, they have been shown to be extremely effective at interpreting the what, i.e. identifying positive events within a dataset 
Figure 3. Minute-wise comparison of event identification stability with respect to $\Delta B_{\theta}$ for

five reconnection catalogues created through consecutive runs of the G21 neural network classifi-

cation algorithm. The probability of variance indicates the fractional variation of identifications

between two runs in a given $0.25 \mathrm{nT} \Delta B_{\theta}$ bin.

on par with or outperforming human classifiers (He et al., 2015; Geirhos et al., 2018). This is why domain knowledge is critical, to marry the computation power of an ML algorithm with the domain expertise for interpretation of the scientific context. Similarly issues of stability are introduced when utilizing ML methods. Machine learning is defined by user set hyper parameters and a random starting configuration which is then fine tuned through successive epochs of training into an effective classifier. Hence, successive runs of a machine learning algorithm can produce varying results, even when using the same hyper parameters, due to the random starting positions of weights and biases, the method of separating train/test datasets, and limitations of epochs of training.

Stability for ML algorithms is typically assessed through a validation of the produced results. Metrics of accuracy and various skill scores are used to indicate an algorithm's performance on a validation or test dataset, a classified dataset which has never been seen by the algorithm during training. This is typically extremely effective at indicating an algorithm's performance and more than justifies its use when extrapolated to larger datasets. However, in space physics we typically operate in less controlled environments, with rare phenomena resulting in class imbalances and unequal spatial and temporal coverage, hence when an algorithm is shown to be effective on a classified subset, it is not indicative of its performance on a larger unclassified dataset that experiences more varied background environments (Schneider et al., 2020). The ML algorithm constructed in G21 was trained, tested and validated on the S16 catalogue which only covers dates from the years 2006, 2009, and 2010. These years are during the optimal Cassini orbits for detecting and identifying magnetotail reconnection bi-products. Extrapolating the catalogue from these years to more varied orbits allows for the identification of more events, however these events cannot be automatically verified. Instead, we can validate these detections by comparing how consistently they are identified for each consecutive run of the ML algorithm. Figure 3 compares the distributions of $\Delta B_{\theta}$ across 5 consecutive runs of the G21 model. These runs were trained with the same hyperparameters, such as number of hidden layers, and nodes, etc., but with variations on the training/test/validation set selection. The hyper parameters of a ML algorithm describe its architecture. Neural network nodes, also known as a neuron, is a computational connective unit that takes a number of weighted inputs and combines them algebraically to create an output. Hidden layers are the layers of neural network nodes between input and output layers that allow for more complex linear algebraic fits to the training dataset and represent more complex physical properties of the dataset. The probability of variance in these plots describe the fractional number of minutes in events that are identified by one run of the model and not the other in $0.25 \mathrm{nT}$ bins. Notably, $>1 \mathrm{nT}$ probability of variance reaches a plateau of $\sim 0.1$ across all comparisons. This indicates that $10 \%$ of detections are likely to vary between runs of the ML model. Similarly, low $\Delta B_{\theta}$ events $(<0.5 \mathrm{nT})$ reach a higher variance of $\sim 0.5$. This indicates that while these detections may represent correctly identified reconnection products, they can't be consistently identified and hence should be excluded from statistical examination.

Determining an optimal threshold, above which we consider events to be validated, is difficult as, while the overall shape of distributions are similar, small scale structure in these distributions have some variation in each run comparison. To remove these local topological variations the distributions of all comparison (excluding comparisons of individual runs with themselves) can be averaged to obtain Figure 4. This figure displays average variability distributions across three parameters observed for each event in the G21 catalogue, namely (a) $\Delta B_{\theta}$, (b) $\Delta t$, and (c) signal to noise ratio. These signal to noise ratio values are calculated in $\mathrm{G} 21$ as: 


\section{Mean variability of reconnection events across the 5 ML runs}

Figure 4. Comparisons of mean stability across the aforementioned five reconnection cat-

alogues. Stability is analyzed using metrics of $\Delta B_{\theta}, \Delta t$, and signal to noise ratio for events.

Events with a variance below a threshold of 0.15 are considered to validated for statistical anal-

ysis. This creates lower limit thresholds for $\Delta B_{\theta}, \Delta t$, and signal to noise ratio for events of

$0.71 \mathrm{nT}, 6.61 \mathrm{~min}$, and 1.15 respectively.

$$
S N R=\frac{\left|\Delta B_{\theta}\right|}{B_{\theta}^{R M S}}
$$

where $B_{\theta}^{R M S}$ is the average for a period extending $30 \mathrm{~min}$ either side of the central time of the event, originally sourced from S16. The distributions shown in these plots are similar to one another with a high level of variance for low values which eventually plateau at $\sim 0.1$ variance. A threshold of $<0.15$ variance is selected as a reasonable confidence interval, below which an event is considered validated. This renders three parametric thresholds of $\Delta B_{\theta}=0.71 \mathrm{nT}, \Delta t=6.61 \mathrm{~min}$, and $\mathrm{SNR}=1.15$. It must be noted, that small-scale drizzle on the day-side has been discussed by (Delamere et al., 2015) as a mechanism for material to be transported out of magnetospheres. Hence, for smaller duration events ( $\Delta t \sim 1$ minute) it would be expected to identify a larger number of candidates on the dayside to dusk flank of the magnetosphere. This is the theoretically expected scale of these events. However, due to physical limitations, our empirical threshold cannot probe identifications of this scale due to a lack of variability observed in Figure 4, and the approach of the length scale of classification to the time resolution of the dataset used for classification. Hence, vents with all parameters above these thresholds are considered to be validated events and will be used to identify statistical trends in reconnection events. The G21 ML algorithm and associated catalogue are publically available at Garton (2020)

\section{Results}

The ML approach is trained to identify magnetic field deflections like those in the training set. This means that bipolar deflections can be selected at any point along the Cassini trajectory (see Figure 2): not just confined to the magnetotail, but including the dayside, as well as even during magnetosheath or solar wind excursions. Figure 5 indicates the number of events identified by the ML algorithm while the Cassini spacecraft was located in the solar wind (dark blue), magnetosheath (light blue), the day-side magnetosphere (light salmon) and the night-side magnetosphere (dark salmon) for all identifications in the catalogue. These magnetic environment classifications are obtained from the Jackman et al. (2019) catalogue of magnetopause and bow shock crossings. Each of the bars in the graph are shaded with the number of events in each region that are above the three limitations set in Figure 4: 1111 solar wind, 9558 magnetosheath, 4128 day-side, and 3472 night-side events respectively. Since this catalogue is constructed from a ML method built upon the S16 catalogue, it is believed that the 3472 events within the night-side magnetosphere are considered confirmed as they represent identifications in a similar environment and under the same magnetic conditions as the catalogue the ML algorithm learned from. This does not indicate that the detections outside of the aforementioned limitations, or outside of the magnetosphere are not true identifications of signatures of reconnection, merely that they cannot be substantially validated. Signals of reconnection have been identified previously in the magnetosheath (Huddleston et al., 1997; Badman et al., 2013), on the magnetopause (Jasinski et al., 2016, 2021), and on the day-side magnetosphere (Delamere et al., 2015; Guo et al., 2018), however the underlying physical mechanisms and magnetic field morphologies which may lead to these bipolar deflections are different. On the nightside, the physical picture developed in the S16 catalogue is one of stretched 
Figure 5. Number of identified events by Cassini during its near-Saturn lifetime in the four magnetic environments: solar wind, in the planetary magnetosheath, day-side, and night-side magnetosphere. Reconnection events were classified into these regions by the Jackman et al. (2019) catalogue. Shaded regions of each classification represent the number of events that meet the thresholding criteria established in Figure 4.

Figure 6. Yearly distribution of identified reconnection events during Cassini's near-Saturn lifetime for all (dark blue), magnetosphere (light blue, and night-side magnetosphere events within limitations established in Figure 4. On average, $\sim 7000$ events are identified yearly by the in-situ spacecraft, however, this does not account for all magnetospheric reconnections as only events that occur upwind of Cassini will be identifiable. Furthermore, this distribution does not account for the varying orbital trajectories of Cassini during its lifetime.

magnetic field lines reconnecting and releasing plasmoids downtail or dipolarizations planetward of the reconnection site. Reconnection in the day-side plasma sheet is likely to have a somewhat different morphology given the confinement by the magnetopause limiting the degree of current sheet stretch. Moreover, reconnection in the turbulent magnetosheath is also a process of likely different character to large-scale magnetotail reconfiguration.

\subsection{Temporal Statistical Analysis}

Figure 6 illustrates the temporal distribution of reconnection identified for the entirety of Cassini's lifetime for all events (dark blue), events within the magnetosphere (light blue), and the aforementioned thresholded events within the night-side magnetosphere (salmon). Notably, the three distributions are dissimilar due to the varying trajectory of Cassini's orbit throughout its lifetime, with the initial capture orbits following Saturn Orbit Insertion in 2004 favouring large radial distance detections (many in the solar wind and magnetosheath), in contrast to detections in 2017 during Cassini's proximal orbits favouring small radial distance identifications (inner to middle magnetosphere). Furthermore, no class of identifications maintains a consistent yearly rate of events due to these varying orbits. Most apparent are an absence of validated events present in 2008 due to Cassini entering a high-latitude polar orbit with small equatorial plane radial distances as indicated in Figure 2. The largest number of validated detections occur in 2006 and 2010 where Cassini entered into deep-tail equatorial orbits where it would be closest to the magnetotail current sheet, the site of reconnection. Hence, these years are likely the most accurate representation of magnetotail reconnection rates with $\sim 900$ yearly identifications. However, even during these orbits, Cassini is located out of these spatial ranges for significant periods of time. Hence, the true number of identification for a spacecraft in an ideal location $\left(N_{\text {total }}\right)$ can be calculated as:

$$
N_{\text {total }}=N_{i} \frac{T_{\text {total }}}{T_{i}}
$$

where $N_{i}$ is number of validated events within limits $(3472 \pm 59), T_{\text {total }}$ is the total time of Cassini's near-Saturn lifetime $\left(6.77 \times 10^{6} \mathrm{mins}\right)$, and $T_{i}$ is the total time where Cassini is within the magnetotail within $\pm 40^{\circ}$ latitude of the equatorial plane $\left(2.48 \times 10^{6} \mathrm{mins}\right)$. These values render an estimation of $N_{\text {total }} \approx 8895 \pm 151$ for Cassini's near-Saturn lifetime, or $1.87 \pm 0.03$ identifications per day. 
Figure 7. Reconnection occurrence as a function of latitude for the 3472 events in KRTP coordinates for the entirety of Cassini's near-Saturn lifetime. Colour in these plots indicate (a) the radial distance of Cassini from Saturn during detection and (b) the number density of detections for given latitudes with time. The majority of reconnection is limited to the planetary current sheet, with variation from the 0 degrees latitude matching the expected long-term seasonal change of the planetary magnetic field.

The structures associated with magnetic reconnection are expected to initiate within the current sheet, where anti-parallel field lines meet (Harris, 1962; Connerney et al., 1983; Arridge et al., 2008b). However, since Saturn's axis has a seasonal tilt as it orbits the Sun and due to its large and expansive magnetosphere, its current sheet is known to become hinged with the seasonal variation of the planet (Arridge et al., 2008a; Carbary et al., 2015). This effect is visible in Figure 7 (a) and (b) which compares the latitude and radius of the aforementioned 3472 events in Kronian Radial-Theta-Phi (KRTP) coordinates across Cassini's lifetime. KRTP coordinates are defined as the polar representation of a Cartesian coordinate system, where the $\mathrm{x}$ axis is positive Sun-ward in the Sun-Saturn line, the $\mathrm{z}$ axis is positive in the direction of Saturn's north magnetic pole, and the y axis completes the right handed set.

In 2004 Saturn experienced southern hemisphere summer, where the current sheet extends perpendicular to the rotational axis of the planet until $\sim 25 \mathrm{R}_{S}$. Beyond this distance, the pressure of the incoming solar wind overcomes the planet's magnetic pressure, causing the magnetotail to be swept out of the plane, creating a hinged magnetotail current sheet (Arridge et al., 2008a, 2011). Notably, an overall preference exists for negative latitude detections in 2004, whereas in 2017 a preference exists for positive latitude detections. This effect is due to this aforementioned current sheet hinging, which varies on the seasonal timescale of Saturn. This phenomenon is visible in the data where the large radial distance identifications occur close to $0^{\circ}$ latitude, while identifications closer to the planet $\left(<30 \mathrm{R}_{S}\right)$ follow the seasonal tilt variation of Saturn. In 2009, Saturn was experiencing an equinox. This means the current sheet is expected to have no hinging at this time, which is reflected by the reduced range of latitudinal detections during this period to being highly localized around $0^{\circ}$. However, the magnetotail current sheet does exhibit vertical flapping of the current sheet, closely linked to the Planetary Period Oscillations (PPOs) [e.g. Bradley et al. (2018)]. This flapping means that the current sheet can reach a modest range of latitude above/below its nominal central position. During 2009 and 2010, Cassini's trajectory was changed to an equatorial orbit with apokrone in the magnetotail at radial distances out to $\sim 50 \mathrm{R}_{S}$, greatly facilitating the identification of magnetotail reconnection events. Other patterns of detections exist in this dataset, namely the extremely high and low latitude detections of 2007, mid 2009, 2013, and 2014. Identifications at these latitudes are due to the highly angled orbital trajectory of Cassini at these times (see Figure 2), where it comes close to the magnetopause boundary and hence may identify Kelvin-Helmholtz (K-H) instabilities in magnetic field observations (Delamere et al., 2013b; Johnson et al., 2014; B. L. Burkholder et al., 2020). To reduce the impact of these detections, identifications within one hour of Cassini crossing the magnetopause were removed from the magnetosphere detections, however it must be noted that global-scale $\mathrm{K}-\mathrm{H}$ vortices may still be identified by the ML algorithms, however, there is no way to sufficiently weed out these detections spatially or temporally without potentially removing valid identification events. When comparing these events with their number density it is apparent that these peculiar events are few in number and hence possibly a simple statistical error. The high latitude bins of 2013/2014 however exhibit $>10$ detections in number density and hence can be considered statistically significant, they are at larger radial distances $\left(>50 \mathrm{R}_{S}\right)$. This may imply these identifications are due to interactions with the magnetopause boundary or its nearby magnetic environment that are not removed by the Jackman et al. (2019) catalogue. This may be due to the spacecraft not crossing the boundary layer (Masters et al., 2011), but still orbiting close enough to be affected by its near plasma environment. 
Figure 8. Radial (a), local time (b), and latitudinal (c) distributions of identified reconnection events for all events (dark blue), only events within the magnetosphere (light blue), and events within the magnetosphere and above the aforementioned parametric thresholds (salmon).

Figure 9. Normalized global distribution of identified reconnection events viewed from three different perspectives in a logarithmic colour scale from three viewpoints $\sim 1600$ (a), $\sim 2000$ (b), and $\sim 0200$ (c) hours local time.

\subsection{Spatial Statistical Analysis}

Figure 8 indicates the spatial distribution of reconnection for all events in the G21 catalogue (dark blue), magnetosphere only (light blue), and night-side magnetosphere within the aforementioned limits (salmon). The distribution of events is described as functions of radial range (a), local time (b), and latitude (c). The majority of reconnection across all classes occurs in the $20-40 \mathrm{R}_{S}$ range, however this is most likely due to Cassini spending much of its lifetime in this radial distance range, showing favour for detection at these distances. The local time distribution of reconnection is highly imbalanced in favour of dusk-side reconnection. This effect is most notable in the magnetospheric class where the number of dawn-side detections can be a factor of two times lower than dusk-side. However this effect still persists in our limited 3472 night-side events, with a high density of observations in the 18-21 hours range where the rotating magnetosphere enters a large scale expansion down tail. The latitudinal distribution of events is highly focused in the equatorial plane, particularly for the limited dataset. Identifications outside of the $20^{\circ}$ wide bin centred on $0^{\circ}$ may be attributed to the observation of plasmoids near a current sheet which has two key physical phenomena controlling its location: (i) the seasonal variation and associated hinging of the current sheet, (ii) the vertical flapping accompanied by quasi-periodic thickening and thinning of the current sheet modulated by the planetary period, e.g. Provan et al. (2018) and references therein. Furthermore, a subset of the nightside detections correspond to TCRs as opposed to plasmoids, with the former being observable from the higher latitude lobes as opposed to the central current sheet region. Moreover, there is an expected lack of events at highest latitudes $\left(> \pm 70^{\circ}\right)$ furthest from the theoretical sites where oppositely directed field lines from opposite hemispheres could merge and reconnect.

Figure 9 demonstrates global distribution of reconnection events within thresholds from Figure 4, but across all local times from three viewpoints at $\sim 1600$ (a), 2000 (b), and $\sim 0200$ (c) hours local time. Night-side events are highly restricted to equatorial latitudes with maximal extents at $\sim 40^{\circ}$, with concentration centered around $0^{\circ}$ latitude. Two relative hotspots exist in these night-side detections corresponding with the number of events at 2000 and 0200 hours local time from Figure 8. Day-side detections are observed to have a far larger latitudinal spread than night-side detections (Neupane et al., 2021). Furthermore, two hotspots exist on the day-side, similar to night-side, however these are located at high latitudes $\left( \pm 40^{\circ}\right)$ and restricted in local time (1400-1600 hours). Since these identifications are on the day-side, where the machine learning algorithm has never been trained on, it is difficult to conclude these detections correspond to true events, however acknowledging them and their potential indication of some physical phenomenon other than reconnection makes them noteworthy observations. Future research into these dayside identifications would require use of a newly constructed ML catalogue designed and trained to be inclusive of day-side reconnection events that may not fit the typical bipolar magnetic signatures observed in night-side reconnection.

Figure 10 demonstrates (a) an equatorial projection distribution of magnetospheric deflections (both day-side and night-side) and (b) the $\left|\Delta B_{\theta}\right|$ of events in these spatial bins. The occurrence distribution is normalized with respect to observation time of Cassini in each spatial bin, hence colour in this plot indicates the probability of identifying a reconnection event for every minute of observation in each spatial bin. Lack of colour in a given 
Figure 10. (a) Normalized radial distribution of identified reconnection events normalized for

Cassini observing times in each spatial bin. (b) Mean $\left|\Delta B_{\theta}\right|$ for identified events in each radial

and local time bins.

sector is attributed to either the Cassini spacecraft not exploring that region during its lifetime, or a lack of field deflection detections (despite Cassini sampling). Three main clusters of reconnection are identifiable in this figure, at $0300-0600,1200-1700$, and 1900 - 0200 hours local time. The 0300 - 0600 local time cluster of reconnection probability is due a normalization effect. Very few detections $(<10)$ exist in these spatial bins however the probability of identification is high due to Cassini occupying these bins for a small period of time, hence a high rate of error is associated with this cluster. Furthermore, these detections are close to the magnetopause boundary and are possibly due to near-boundary interactions. The cluster at $1200-1700$ hours is associated with the two day-side hotspots of identifications discussed for Figure 9. Finally, the cluster of detections from 1900 - 0200 hours local time occur in statistically significant numbers and encompass a local time sector which was well sampled in the training data. This cluster represents the preferential region for identifying tail-side reconnection signatures. Unfortunately, the Cassini spacecraft has a lack of observations directly in the center of this cluster at $\sim 2100$ local time beyond $35 \mathrm{R}_{S}$. This cluster may be far more populated with reconnection signatures if Cassini's trajectory had entered these spatial bins. Even with the lack of observations in these spatial bins it is still clear that a significant imbalance for detection exists in favour of dusk-side identifications. The $\left|\Delta B_{\theta}\right|$ plot shows a preference for larger deflection events to occur closer to the planet. This is likely due to magnetic field strength being stronger closer to the planet and typical signatures of deflections featuring a polarity inversion. Hence, for a typical reconnection event, a larger $\Delta B_{\theta}$ deflection would be expected closer to the planet. Also of note in this plot are the asymmetries between day and night-side, and dawn and dusk. Day-side show overall larger deflections than night-side for the same radial distances. This may be due to the unverified nature of these day-side identifications. It's possible that day-side reconnection facilitates the creation of stronger magnetic features. Alternatively it may be that due to this environment being so different to the night-side events the model was trained to identify, it can only consistently identify the larger magnetic deflection signatures. The dawn-dusk asymmetry is approximately inverse of the reconnection occurrence distribution. This may mean the apparent stronger magnetic signatures on the dawn side are a feature of uncertainty, or alternatively, it may imply that there is no asymmetry in flux transport for Saturn, merely that on the duskside, flux is transported often on small scale and that on the dawn-side flux is transported less frequently but in larger scales. Interestingly, this figure matches well with the heating rate densities due to turbulent processes. Turbulent heating rates are determined by magnetic fluctuations (Kaminker et al., 2017), and the local time asymmetry of this reconnection rate distribution is being similar to that of the turbulent heating densities at Saturn is further evidence between the linking of the two.

Figure 11 indicates the directionality of the 3472 validated magnetotail events normalized with respect to the mean number of observations in each $2.5 \mathrm{R}_{S}$ bins. This normalization method better represents the directionality of events at a given radial distance for the imbalanced number of observations in both directions. The planetward (1162) and tailward (2310) classifications come directly from the G21 catalogue and can be inferred where positive to negative $\Delta B_{\theta}$ events are considered to be planetward and tailward events exhibit a negative to positive $\Delta B_{\theta}$ deflection. Notably, planetward event occur at lower radial distances on average $\left(32.8 \mathrm{R}_{S}\right)$ than tailward events $\left(34.7 \mathrm{R}_{S}\right)$, however a significant overlap exists between the two distributions making it difficult to distinctly classify them and hence identify the location of a planetary x-line.

\subsection{Parametric Statistical Analysis}

The G21 catalogue extracts parametric information for each identified event. Figure 12 demonstrates the distribution of duration for the aforementioned 3472 events in 
Figure 11. Radial distribution of planetward (blue) and tailward (red) events for night side

identifications. Notably, planetward identifications are observed on average closer to the planet

(Mean at $32.8 \mathrm{R}_{S}$ ) than tailward events (Mean at $34.7 \mathrm{R}_{S}$ ), however there is a significant overlap of the two distributions.

Figure 12. Log-log distribution of identified event duration for events longer than the aforementioned stability thresholds. This distribution is assumed to be exponential, with the implication being reconnection events are scale invariant, and is described by the equation: $N=299.23 \times \Delta t^{-1.28}$.

log-log space, normalized to give the expected number of detection in each $\Delta t$ bin in a single year. This method enables a first approximation of the size and frequency of events expected to be observed by an in-situ spacecraft, however, the visibility of plasmoids is a function of the spacecraft proximity to the center of the current sheet, which itself is variable depending on current sheet flapping and wave motion (Jackman et al., 2009; Andrews et al., 2019). Delamere et al. (2015) investigated the time between consecutive current sheet crossings by Cassini and found a similar shaped, power-law-like distribution. Under the assumption of a scale invariant or fractal-like nature of the planetary magnetotail (Hoshino et al., 1994; Milovanov et al., 1996; Bradley et al., 2018), a power law relationship is fit to the distribution $\left(N=299.23 \times \Delta t^{-1.28}\right)$. This fit performs well for mid duration events in this distribution, however both the long duration and short duration events are observed to fall below this fit. This is likely caused by the potential missing of small scale events due to their relatively lower signal to noise ratio and their approaching of the time resolution of the dataset, thus being more susceptible to missed classifications. Long duration events are significantly rarer, and thus the chance they are not observed due to the spacecraft not being in the optimal observing window when they occur has a higher impact on their statistical distribution. Alternatively, a different fitting function may be more optimal to fit the distribution, however this would require an assumption of scale variance for reconnection events, whereas assuming the catalogue misses some difficult to identify events is more probable. From this fit, it can be estimated that a spacecraft is expected to observe $\sim 307$ one minute duration events every year, however since these events (1) occur on such a short time scale, and (2) are likely to be very small compared to background magnetic topology, it is likely that these events will not by identified within magnetic field observations; however they provide a rough estimate of number and size of events for approximating mass loss for Saturn's magnetosphere. Similarly, extremely large and rare events can be approximated, for example a one in ten year event would have a yearly observation rate of $N=1 / 10$, and from our power law fit, a duration of $\sim 459$ minutes. It is important to note that while this fit provides a potentially infinite size scale for reconnection events, in reality these events are limited in duration due to the finite scale of the magnetosphere and limitations on factors like flux tube content, inflow to the diffusion region (Goertz, 1983; Arridge et al., 2015). Some upper limit of duration exists, however during the 13 years of Cassini's observations, not enough reconnection events were detected to statistically conclude this upper limit.

Figure 13 demonstrates a similar parametric distribution of $\Delta B_{\theta}$ for the 3472 events in log-log space, normalized to a year timescale. This distribution is also well described by a power law relationship $\left(N=52.07 \times \Delta B_{\theta}^{-2.08}\right)$. This distribution enables a similar level of predictability to reconnection event scale. The power law fit supports the idea for a fractal structure/scale invariant mechanism behind the creation of reconnection signatures. Furthermore, with this distribution it is possible to estimate yearly event occurrence for given $\Delta B_{\theta}$ bins. Hence, it is expected to observe $\sim 50$ events with $0.875<\Delta B_{\theta}<1.125$ and it is expected to observe a single $\sim 16 \mathrm{nT}$ deflection event every ten years. It must be remembered that this distribution is also subject to the spatial limitations of the Kronian magnetosphere and some upper limit of $\Delta B_{\theta}$ exists that cannot be statistically identified 
Figure 13. Log-log distribution of identified event north-south magnetic deflection for events

above the aforementioned stability thresholds. Assuming these events are scale invariant, an exponential line is fit to the distribution, of the order $N=52.07 \times \Delta B_{\theta}^{-2.08}$.

exclusively using Cassini's observations. For example, plasmoids can only be as wide as the total magnetotail width $\left(\sim 90 \mathrm{R}_{S}\right)$. In actuality, it is likely typical plasmoid sizes don't approach these widths. This is inferred from research on relative sizes of plasmoids in Earth's magnetosphere (Ieda et al., 1998). Plasmoids are created within the current sheet which itself has constraints on vertical extent [range 1-6 $\mathrm{R}_{S}$, Giampieri and Dougherty (2004); Dougherty et al. (2005); Arridge et al. (2008b); Staniland et al. (2020)]. In practice, plasmoids represent localized bulges in the plasma sheet (as evidenced with the observation of TCRs) but there is a limit on this deformation of the current sheet. This creates strict spatial limitations on plasmoids. Similarly, $\Delta B_{\theta}$ is limited by the available magnetic flux of Saturn, which is typically observed through variation in the auroral oval (Badman et al., 2005; Carbary, 2012; Badman et al., 2014). For this research, we assume that the spacecraft travels directly through the center of the identified plasmoid in a head-on trajectory, hence causing the maximum possible $\Delta B_{\theta}$ delflection. In reality, it is likely the spacecraft intersects the majority of plasmoids in a glancing blow or off center trajectory causing a smaller observed $\Delta B_{\theta}$.

\section{Discussion}

As evidenced in the previous section, the ML catalogue created by G21 opens the path for statistical studies on the properties of magnetospheric reconnection for Saturn. This research has focused on a small, spatially restricted, validated dataset, and exclusively on the magnetic field properties as measured by Cassini.

From the temporal statistical analysis, we can conclude that spacecraft orbiting Saturn may sample $\sim 200$ magnetotail reconnection events every year. Spacecraft in deep-tail orbits are more likely to experience higher reconnection rates of $\sim 900$ events. However, through normalizing the total number of validated detections across Cassini's lifetime (3472) with respect to time Cassini spent in the optimal spatial window for observing magnetotail reconnection, an estimate of $\sim \quad 660$ events occur yearly. This knowledge is crucial for establishing expectations for future spacecraft missions to Saturn. Furthermore, it can be concluded the seasonal variation and hinging of the current sheet for Saturn can be identified by analysing the locations of reconnection identification for tail-side events. While this dataset only covers $\sim 14$ years (less than half of the orbital period of Saturn), the latitudinal variation of detections follows closely the seasonal tilt of Saturn's magnetosphere. For the near current sheet latitudinal identifications, large radial distance observations occur more closely to $0^{\circ}$, while closer detections follow the planet's seasonal tilt. The shift between these two regimes occurs in the range of 20-30 $\mathrm{R}_{S}$ indicating a hinge in the planetary current sheet at $\sim 25 \mathrm{R}_{S}$ which agrees with the theoretical location of the current sheet hinge for Saturn (Arridge et al., 2008a; Carbary et al., 2015). Notably, these findings are inclusive of plasmoids, TCRs, and dipolarizations. While plasmoids and TCR events are well constrained to the current sheet, dipolarizations are not and hence may have a wider latitudinal spread, hence causing near Saturn events $\left(<30 \mathrm{R}_{S}\right)$ to be observed at higher latitudes.

From the spatial distribution of events, it can be concluded that night-side magnetospheric reconnection signatures are most identified in the 20-40 $\mathrm{R}_{S}$ range, with a preference for the identification of many small-scale events in the dusk-side of the magnetosphere. This may imply a preference for Vasyliunas style reconnection over Dungey cycle style (Badman \& Cowley, 2007). Vasyliunas reconnection is associated with mass loss and Dungey cycle reconnection is associated with both mass loss and flux closure. Hence, this preference for Vasyliunas style reconnection favours a loss of mass with no change in the ratio of open and closed magnetic fields. Furthermore, observations of reconnec- 
tion are localized to the equatorial plane, particularly for night-side reconnection. This can be explained as reconnection occurs localized within the planetary current sheet and signatures of reconnection travelling along the current sheet. Also of note, dayside field deflections from the catalogue, while not validated, seem to be observed across a broader range of latitude and longitude with notable hotspots at $\pm 40^{\circ}$. These hotspots conform to the non-equatorial disturbed magnetic field events of (Neupane et al., 2021), however, they observed a more uniform distribution with latitude. Alternatively, these hotspots may be the most prominent location for magnetic flux tube twisting that facilitates the double reconnection process at mid latitudes from (Ma et al., 2019). The precise cause of these hotspots, and their link to specific magnetospheric phenomena would be an interesting avenue for future research on dayside processes.

From the research on directionality of reconnection events and the highly overlapped distribution of tailward and planetward events, it is concluded that either the planetary $\mathrm{x}$-line is highly mobile across the 14 year period, or that its location is beyond Cassini's observing window for much of the time. Previous studies at the gas giants have sought to explore the x-line location and properties. Vogt et al. (2010) examined Galileo data at Jupiter and found a reasonably clear, statistically significant boundary between tailward and planetward events, indicating a jovian tail x-line at $\sim 90 \mathrm{R}_{J}$ at dawn, and $\sim 120 \mathrm{R}_{J}$ at dusk. More recently, Vogt et al. (2020) took a similar approach to event identification and performed a statistical analysis of Juno magnetometer data. That study did not reveal a statistical x-line position. Thomsen et al. (2014) studied plasma flow parameters at Saturn and found that no quasi-steady $\mathrm{x}$-line position was found within $45 \mathrm{R}_{S}$. From Figure 11 an unstable equilibrium point may exist at $\sim 33.75 \mathrm{R}_{S}$ that the $\mathrm{x}$-line will tend to on average, however it is likely that due to the variable nature of Saturn's magnetic field and the surrounding solar wind, the location of the x-line is equally as variable (Jia et al., 2012; Smith et al., 2016, 2018a). The coverage of the terrestrial magnetotail allows for examination of both the near-Earth and the distant tail x-line (Ieda et al., 1998; Eastwood et al., 2005; Imber et al., 2011). Jackman and Arridge (2011b) noted that the down-tail coverage at Jupiter and Saturn equates to $\sim<3$ times the typical magnetopause standoff distance at those planets, whereas the coverage at Earth with spacecraft like Geotail $\sim 200 \mathrm{R}_{E}$ equates to $\sim 20$ times the typical terrestrial standoff distance. Thus the exploration space at the gas giants is much more limited. By applying simple scaling from Earth to account for the planetary magnetic field strength, the magnetopause standoff distance, and the observed terrestrial near-planet $\mathrm{x}$-line location, one might expect Saturn's x-line to lie $\sim 75 \mathrm{R}_{S}$ from the planet on average (Jackman et al., 2014). However, it must be noted that Earth's magnetosphere is Dungey cycle dominated and hence its phenomenon may not be directly transferable in this manner. Since Cassini spent the majority of observations planetwards of this distance, this theoretical location cannot be confirmed with this research.

From the parametric distribution of events, it is concluded that Saturn's magnetotail has a fractal-like nature for reconnection, i.e. the same underlying processes create both large and small events with an inverse power law distribution of occurrence. This type of distribution is similar to scale size found for magnetohydrodynamic modelling of magnetic islands (Fermo et al., 2010), and in the size distributions of reconnection products in other magnetospheres (Fermo et al., 2011; Akhavan-Tafti et al., 2018; Smith et al., 2018c). This relationship is true for both the duration $(\Delta t)$ and the deflection in north-ward magnetic field $\left(\Delta B_{\theta}\right)$. In Section 3.1, the reconnection rate for Saturn's magnetotail was found to be $\sim \quad 1.87$ reconnection events per day, however this only accounts for events above our statistical thresholds $(\Delta t>6.61 \mathrm{mins})$. From the distribution of $\Delta t$, this reconnection rate can be corrected to include small events through solving the following integrals:

$$
\begin{gathered}
1.87=c \int_{6.61}^{\infty} 299.23 \Delta t^{-1.28} d \Delta t \\
R R_{\text {corr }}=c \int_{1}^{\infty} 299.23 \Delta t^{-1.28} d \Delta t
\end{gathered}
$$

where $c$ is a constant that accounts for converting the fit from yearly events to daily events, and also corrects for the time the spacecraft spent in the optimal viewing position relative to the time of its entire orbit as mentioned in Section 3.1. $R R_{\text {corr }}$ is the true reconnection rate for plasmoids and is calculated to be $3.21 \pm 1.57$ reconnection events per day. 
Similarly, from the $\Delta t$ distribution and previous studies of plasmoid properties, it is possible to estimate the mass loss in Saturn's magnetotail as:

$$
\begin{gathered}
M=\rho V \\
M=\rho W H v \Delta t
\end{gathered}
$$

where $\rho$ represents the density of the plasmoid $\left(0.1 \mathrm{~cm}^{-3}\right.$ of $16 \mathrm{amu}$ ions; Thomsen et al. (2014)), $V$ is the volume of the plasmoid (assumed to be a cuboid for simplicity), $W$ is the width of the plasmoid (ranging in size from $3 \mathrm{R}_{S}$ lower limit Smith et al. (2018a) to $90 \mathrm{R}_{S}$ upper limit at full tail width; Jackman et al. (2014)), $H$ is the height of the plasmoid (4 $R_{S}$; Kellett et al. (2009); Arridge et al. (2011); Sergis et al. (2011); Szego et al. (2012); Staniland et al. (2020)), and $v$ is the velocity of the moving plasmoid $\left(300 \mathrm{~km} \mathrm{~s}^{-1}\right.$; Jackman et al. (2014)). Inserting these values and solving for $M$ gives:

$$
M=524.31 \Delta t
$$

where $\Delta t$ is in seconds. By taking the duration distribution of identified events from Figure 12 , with a maximum observed duration of 400 minutes (from the G21 catalogue) as the upper limit on length $\left(L=v \Delta t \approx 120 \mathrm{R}_{S}\right)$, the mean mass per plasmoid can be calculated as $1.20 \times 10^{6}\left( \pm 2.48 \times 10^{5}\right) \mathrm{kg}$. Taking the aforementioned reconnection rate of $3.22 \pm 1.57$, this gives an estimate of the minimum to maximum of mass loss through plasmoids of $1.50( \pm 0.79) \quad-\quad 44.87( \pm 23.76) \mathrm{kg} s^{-1}$. This finding aligns with previous estimates of Kronian plasmoid mass and mass loss rates (Bagenal \& Delamere, 2011; Jackman et al., 2014). Comparing this mass loss rate to the mass loading rate from Enceledus (8-250 $\left.\mathrm{kg} \mathrm{s}^{-1}\right)$ suggests a sizeable role for a viscous like interaction at Saturn (Delamere \& Bagenal, 2013a; Delamere et al., 2018).

The G21 catalogue opens a new avenue for planetary magnetospheric research by providing the most comprehensive catalogue of magnetic field deflections in the Saturn system, covering 14 years of Cassini data, different Saturn seasons and an entire solar cycle. This paper provides an investigation of reconnection events identified within, with a heavy focus on night-side magnetospheric activity. However, this research can be built upon to investigate signatures of day-side reconnection, or for the events located in the magnetosheath or solar wind. Investigations into all identified events along with a comparison of plasma properties from the CAPS plasma spectrometer may render further understanding of reconnection, and bulk plasma flow within the magnetosphere. Additionally, much of this research has focused on identifications from a model trained on bipolar field signatures observed in the magnetotail. For future work that aims to focus on the dayside or the dawn-dusk asymmetry in magnetospheric reconfiguration, it would be prudent to train an algorithm specifically on a labelled dataset from those regions. Finally, the ML method applied to the Cassini observations may be expanded, and retrained for missions that have focused on other planets in our solar system such as MESSENGER at Mercury, and Galileo or Juno at Jupiter.

\section{Data Availability Statement}

Calibrated data from the Cassini mission are available from the NASA Planetary Data System at the Jet Propulsion Laboratory [https://pds.jpl.nasa.gov/].

The datasets created from this study can be found on Zenodo [DOI: 10.5281/zenodo.4638961].

\section{Acknowledgments}

T.M.G.'s work is supported by the Science and Technology Facilities Council Opportunities Fund Grant ST/T002255/1 and by the Alan Turing Institute Grant EP/N510129/1. 
C. M. J.'s work is supported by the Science Foundation Ireland Grant 18/FRL/6199.

A. W. S is supported by STFC Consolidated Grant ST/S000240/1 and NERC grant $\mathrm{NE} / \mathrm{P} 017150 / 1$

\section{References}

Akhavan-Tafti, M., Slavin, J. A., Le, G., Eastwood, J. P., Strangeway, R. J., Russell, C. T., ... Burch, J. L. (2018). MMS examination of FTEs at the Earth's subsolar magnetopause. Journal of Geophysical Research: Space Physics, 123(2), 1224-1241. Retrieved from https://agupubs.onlinelibrary .wiley.com/doi/abs/10.1002/2017JA024681 doi: https://doi.org/10.1002/ 2017JA024681

Andrews, D. J., Cowley, S. W. H., Provan, G., Hunt, G. J., Hadid, L. Z., Morooka, M. W., \& Wahlund, J.-E. (2019). The structure of planetary period oscillations in saturn's equatorial magnetosphere: Results from the cassini mission. Journal of Geophysical Research: Space Physics, 124(11), 8361-8395. Retrieved from https://agupubs.onlinelibrary.wiley.com/doi/abs/ 10.1029/2019JA026804 doi: https://doi.org/10.1029/2019JA026804

Arridge, C. S., André, N., Khurana, K. K., Russell, C. T., Cowley, S. W. H., Provan, G., .. Young, D. T. (2011). Periodic motion of Saturn's nightside plasma sheet. Journal of Geophysical Research: Space Physics, 116(A11). Retrieved from https://agupubs.onlinelibrary.wiley.com/doi/abs/10.1029/ 2011JA016827 doi: https://doi.org/10.1029/2011JA016827

Arridge, C. S., Eastwood, J. P., Jackman, C. M., Poh, G.-K., Slavin, J. A., Thomsen, M. F., ... et al. (2015). Cassini in situ observations of long-duration magnetic reconnection in Saturn's magnetotail. Nature Physics, 12(3), 268-271. Retrieved from http://dx.doi.org/10.1038/nphys3565 doi: $10.1038 /$ nphys 3565

Arridge, C. S., Khurana, K. K., Russell, C. T., Southwood, D. J., Achilleos, N., Dougherty, M. K., .. Leinweber, H. K. (2008a). Warping of Saturn's magnetospheric and magnetotail current sheets. Journal of Geophysical Research: Space Physics, 113(A8). Retrieved from https:// agupubs.onlinelibrary.wiley.com/doi/abs/10.1029/2007JA012963 doi: https://doi.org/10.1029/2007JA012963 
Arridge, C. S., Russell, C. T., Khurana, K. K., Achilleos, N., Cowley, S. W. H., Dougherty, M. K., .. Bunce, E. J. (2008b). Saturn's magnetodisc current sheet. Journal of Geophysical Research: Space Physics, 113(A4). Retrieved from https://agupubs.onlinelibrary.wiley.com/doi/abs/10.1029/ 2007JA012540 doi: https://doi.org/10.1029/2007JA012540

Azari, A. R., Biersteker, J. B., Dewey, R. M., Doran, G., Forsberg, E. J., Harris, C. D. K., .. Ruhunusiri, S. (2020). Integrating machine learning for planetary science: Perspectives for the next decade. White Paper Submitted to the Decadal Survey on Planetary Science and Astrobiology 2023-2032. Retrieved from https://arxiv.org/abs/2007.15129

Bader, A., Badman, S. V., Yao, Z. H., Kinrade, J., \& Pryor, W. R. (2019) Observations of continuous quasiperiodic auroral pulsations on Saturn in high time-resolution UV auroral imagery. Journal of Geophysical Research: Space Physics, 124(4), 2451-2465. Retrieved from https:// agupubs.onlinelibrary.wiley.com/doi/abs/10.1029/2018JA026320 doi: https://doi.org/10.1029/2018JA026320

Badman, S. V., Bonfond, B., Fujimoto, M., Gray, R. L., Kasaba, Y., Kasahara, S., ... Yoshioka, K. (2016). Weakening of Jupiter's main auroral emission during January 2014. Geophysical Research Letters, 43(3), 988-997. Retrieved from https://agupubs.onlinelibrary.wiley.com/doi/abs/10.1002/ 2015GL067366 doi: https://doi.org/10.1002/2015GL067366

Badman, S. V., Bunce, E. J., Clarke, J. T., Cowley, S. W. H., Gérard, J.-C., Grodent, D., \& Milan, S. E. (2005). Open flux estimates in Saturn's magnetosphere during the January 2004 Cassini-HST campaign, and implications for reconnection rates. Journal of Geophysical Research: Space Physics, 110(A11). Retrieved from https://agupubs.onlinelibrary.wiley.com/doi/abs/ 10.1029/2005JA011240 doi: https://doi.org/10.1029/2005JA011240

Badman, S. V., \& Cowley, S. W. H. (2007). Significance of Dungey-cycle flows in Jupiter's and Saturn's magnetospheres, and their identification on closed equatorial field lines. $\quad$ Annales Geophysicae, 25(4), 941-951. doi: 10.5194/angeo-25-941-2007

Badman, S. V., Jackman, C. M., Nichols, J. D., Clarke, J. T., \& Gérard, J.-C. (2014). Open flux in Saturn's magnetosphere. Icarus, 231, 137-145. Re- 
trieved from https://www.sciencedirect.com/science/article/pii/ S0019103513005137 doi: https://doi.org/10.1016/j.icarus.2013.12.004

Badman, S. V., Masters, A., Hasegawa, H., Fujimoto, M., Radioti, A., Grodent, D., .. Coates, A. (2013). Bursty magnetic reconnection at Saturn's magnetopause. Geophysical Research Letters, 40(6), 1027-1031. Retrieved from https://agupubs.onlinelibrary.wiley.com/doi/abs/10.1002/grl.50199 doi: https://doi.org/10.1002/grl.50199

Bagenal, F., \& Delamere, P. A. (2011). Flow of mass and energy in the magnetospheres of Jupiter and Saturn. Journal of Geophysical Research: Space Physics, 116(A5). Retrieved from https://agupubs.onlinelibrary.wiley .com/doi/abs/10.1029/2010JA016294 doi: https://doi.org/10.1029/ 2010JA016294

Bradley, T. J., Cowley, S. W. H., Bunce, E. J., Smith, A. W., Jackman, C. M., \& Provan, G. (2018). Planetary period modulation of reconnection bursts in Saturn's magnetotail. Journal of Geophysical Research: Space Physics, 123(11), 9476-9507. Retrieved from https://agupubs.onlinelibrary .wiley.com/doi/abs/10.1029/2018JA025932 doi: https://doi.org/10.1029/ 2018JA025932

Bunce, E. J., Cowley, S. W. H., Wright, D. M., Coates, A. J., Dougherty, M. K., Krupp, N., .. Rymer, A. M. (2005). In situ observations of a solar wind compression-induced hot plasma injection in Saturn's tail. Geophysical Research Letters, 32(20). $\quad$ Retrieved from https://agupubs.onlinelibrary .wiley.com/doi/abs/10.1029/2005GL022888 doi: 10.1029/2005GL022888

Burkholder, B., Delamere, P. A., Ma, X., Thomsen, M. F., Wilson, R. J., \& Bagenal, F. (2017). Local time asymmetry of saturn's magnetosheath flows. Geophysical Research Letters, 44(12), 5877-5883. Retrieved from https:// agupubs.onlinelibrary.wiley.com/doi/abs/10.1002/2017GL073031 doi: https://doi.org/10.1002/2017GL073031

Burkholder, B. L., Delamere, P. A., Johnson, J. R., \& Ng, C.-S. (2020). Identifying active Kelvin-Helmholtz vortices on Saturn's magnetopause boundary. Geophysical Research Letters, 47(1), e2019GL084206. Retrieved from https:// agupubs.onlinelibrary.wiley.com/doi/abs/10.1029/2019GL084206 (e2019GL084206 10.1029/2019GL084206)～doi: https://doi.org/10.1029/ 


\section{GL084206}

Carbary, J. F. (2012). The morphology of Saturn's ultraviolet aurora. Journal of Geophysical Research: Space Physics, 117(A6). Retrieved from https:// agupubs.onlinelibrary.wiley.com/doi/abs/10.1029/2012JA017670 doi: https://doi.org/10.1029/2012JA017670

Carbary, J. F., Sergis, N., Mitchell, D. G., \& Krupp, N. (2015). Saturn's hinge parameter from Cassini magnetotail passes in 2013-2014. Journal of Geophysical Research: Space Physics, 120(6), 4438-4445. Retrieved from https://agupubs .onlinelibrary.wiley.com/doi/abs/10.1002/2015JA021152 doi: https:// doi.org/10.1002/2015JA021152

Chen, Y., Hill, T. W., Rymer, A. M., \& Wilson, R. J. $\quad$ (2010). Rate of radial transport of plasma in Saturn's inner magnetosphere. Journal of Geophysical Research: Space Physics, 115(A10). Retrieved from https:// agupubs.onlinelibrary.wiley.com/doi/abs/10.1029/2010JA015412 doi: https://doi.org/10.1029/2010JA015412

Connerney, J. E. P., Acuña, M. H., \& Ness, N. F. (1983). Currents in Saturn's magnetosphere. Journal of Geophysical Research: Space Physics, 88(A11), 87798789. Retrieved from https://agupubs.onlinelibrary.wiley.com/doi/abs/ 10.1029/JA088iA11p08779 doi: https://doi.org/10.1029/JA088iA11p08779

Delamere, P. A., \& Bagenal, F. (2013a). Magnetotail structure of the giant magnetospheres: Implications of the viscous interaction with the solar wind. Journal of Geophysical Research: Space Physics, 118(11), 7045-7053. Retrieved from https://agupubs.onlinelibrary.wiley.com/doi/abs/10.1002/ 2013JA019179 doi: https://doi.org/10.1002/2013JA019179

Delamere, P. A., Burkholder, B., \& Ma, X. (2018). Three-dimensional hybrid simulation of viscous-like processes at Saturn's magnetopause boundary.

Geophysical Research Letters, 45(16), 7901-7908. Retrieved from https:// agupubs.onlinelibrary.wiley.com/doi/abs/10.1029/2018GL078922 doi: https://doi.org/10.1029/2018GL078922

Delamere, P. A., Otto, A., Ma, X., Bagenal, F., \& Wilson, R. J. (2015). Magnetic flux circulation in the rotationally driven giant magnetospheres. Journal of Geophysical Research: Space Physics, 120(6), 4229-4245. Retrieved from https://agupubs.onlinelibrary.wiley.com/doi/abs/10.1002/ 
2015JA021036 doi: https://doi.org/10.1002/2015JA021036

Delamere, P. A., Wilson, R. J., Eriksson, S., \& Bagenal, F. (2013b). Magnetic signatures of Kelvin-Helmholtz vortices on Saturn's magnetopause: Global survey. Journal of Geophysical Research: Space Physics, 118(1), 393-404. Retrieved from https://agupubs.onlinelibrary.wiley.com/doi/abs/ 10.1029/2012JA018197 doi: https://doi.org/10.1029/2012JA018197

Dougherty, M. K., Achilleos, N., Andre, N., Arridge, C. S., Balogh, A., Bertucci, C., ... Tsurutani, B. T. (2005). Cassini magnetometer observations during Saturn orbit insertion. Science, 307(5713), 1266-1270. Retrieved from https://science.sciencemag.org/content/307/5713/1266 doi: 10.1126/science.1106098

Dungey, J. W. (1961). Interplanetary magnetic field and the auroral zones. Phys. Rev. Lett., 6, 47-48. Retrieved from https://link.aps.org/doi/10.1103/ PhysRevLett.6.47 doi: 10.1103/PhysRevLett.6.47

Dungey, J. W. (1965). The Length of the Magnetospheric Tail. Journal of Geophysical Research, 70(7), 1753-1753. doi: 10.1029/JZ070i007p01753

Eastwood, J. P., Sibeck, D. G., Slavin, J. A., Goldstein, M. L., Lavraud, B., Sitnov, M., .. Dandouras, I. (2005). Observations of multiple xline structure in the Earth's magnetotail current sheet: A Cluster case study. Geophysical Research Letters, 32(11). Retrieved from https:// agupubs.onlinelibrary.wiley.com/doi/abs/10.1029/2005GL022509 doi: https://doi.org/10.1029/2005GL022509

Fermo, R. L., Drake, J. F., \& Swisdak, M. (2010). A statistical model of magnetic islands in a current layer. Physics of Plasmas, 17(1), 010702. Retrieved from https://doi.org/10.1063/1.3286437 doi: 10.1063/1.3286437

Fermo, R. L., Drake, J. F., Swisdak, M., \& Hwang, K.-J. ～(2011). Comparison of a statistical model for magnetic islands in large current layers with Hall MHD simulations and Cluster FTE observations.

Journal of Geophysical Research: Space Physics, 116(A9).

Retrieved from https:// agupubs.onlinelibrary.wiley.com/doi/abs/10.1029/2010JA016271 doi: https://doi.org/10.1029/2010JA016271

Fleshman, B. L., Delamere, P. A., \& Bagenal, F. (2010). The Source of Saturn's Extended Neutral Cloud. In Agu fall meeting abstracts (Vol. 2010, p. SM11C- 
1768).

Garton, T. M. (2020). GartontT/SaturnML: Updated catalogue (Version 1.0). Zenodo. Retrieved from https://doi.org/10.5281/zenodo.4638961 doi: 10 $.5281 /$ zenodo.4638961

Garton, T. M., Jackman, C. M., Smith, A. W., Yeakel, K. L., Maloney, S. A., \& Vandegriff, J. (2021). Machine learning applications to kronian magnetospheric reconnection classification. Frontiers in Astronomy and Space Sciences, 7, 104. Retrieved from https://www.frontiersin.org/article/10.3389/ fspas.2020.600031 doi: 10.3389/fspas.2020.600031

Geirhos, R., Janssen, D. H. J., Schütt, H. H., Rauber, J., Bethge, M., \& Wichmann, F. A. (2018). Comparing deep neural networks against humans: object recognition when the signal gets weaker.

Giampieri, G., \& Dougherty, M. K. (2004). Modelling of the ring current in Saturn's magnetosphere. Annales Geophysicae, 22(2), 653-659. Retrieved from https://angeo. copernicus.org/articles/22/653/2004/ doi: 10.5194/angeo-22-653-2004

Goertz, C. K. (1983). Detached plasma in Saturn's front side magnetosphere. Geophysical Research Letters, 10(6), 455-458. Retrieved from https:// agupubs . onlinelibrary .wiley.com/doi/abs/10.1029/GL010i006p00455 doi: https://doi.org/10.1029/GL010i006p00455

Guo, R., Yao, Z., Wei, Y., Ray, L., Rae, I., Arridge, C., .. Dougherty, M. (2018). Rotationally driven magnetic reconnection in Saturn's dayside. Nature Astronomy, 2. doi: 10.1038/s41550-018-0461-9

Harris, E. G. (1962). On a plasma sheath separating regions of oppositely directed magnetic field. Il Nuovo Cimento (1955-1965), 23(1), 115-121.

He, K., Zhang, X., Ren, S., \& Sun, J. (2015). Delving deep into rectifiers: Surpassing human-level performance on ImageNet classification. In Proceedings of the 2015 ieee international conference on computer vision (iccv) (p. 1026-1034). USA: IEEE Computer Society. Retrieved from https://doi.org/10.1109/ ICCV. 2015.123 doi: 10.1109/ICCV.2015.123

Hesse, M., \& Cassak, P. A. (2020). Magnetic reconnection in the space sciences: Past, present, and future. Journal of Geophysical Research: Space Physics, 125(2), e2018JA025935.

Retrieved from https:// 
agupubs.onlinelibrary.wiley.com/doi/abs/10.1029/2018JA025935

(e2018JA025935 2018JA025935) doi: https://doi.org/10.1029/2018JA025935

Hill, T. W., Thomsen, M. F., Henderson, M. G., Tokar, R. L., Coates, A. J., McAndrews, H. J., .. Young, D. T. (2008). Plasmoids in Saturn's magnetotail. Journal of Geophysical Research: Space Physics, 113(A1). Retrieved from https://agupubs.onlinelibrary.wiley.com/doi/abs/10.1029/ 2007JA012626 doi: 10.1029/2007JA012626

Hones, J., E. W. (1977). Substorm processes in the magnetotail: Comments on 'On hot tenuous plasmas, fireballs, and boundary layers in the Earth's magnetotail' by L. A. Frank, K. L. Ackerson, and R. P. Lepping. Journal of Geophysical Research, 82(35), 5633. doi: 10.1029/JA082i035p05633

Hoshino, M., Nishida, A., Yamamoto, T., \& Kokubun, S. (1994). Turbulent magnetic field in the distant magnetotail: Bottom-up process of plasmoid formation? Geophysical Research Letters, 21(25), 2935-2938. Retrieved from https://agupubs .onlinelibrary .wiley.com/doi/abs/10.1029/94GL02094 doi: https://doi.org/10.1029/94GL02094

Huang, S., Zhao, P., He, J., Yuan, Z., Zhou, M., Fu, H., .. Burch, J. (2018). A new method to identify flux ropes in space plasmas. Annales Geophysicae, 36(5), 1275-1283. Retrieved from https://angeo.copernicus.org/articles/36/ 1275/2018/ doi: 10.5194/angeo-36-1275-2018

Huddleston, D. E., Russell, C. T., Le, G., \& Szabo, A. (1997). Magnetopause structure and the role of reconnection at the outer planets. Journal of Geophysical Research: Space Physic, 102(A11), 24289-24004. doi: 10.1029/97JA02416

Ieda, A., Machida, S., Mukai, T., Saito, Y., Yamamoto, T., Nishida, A., ...

Kokubun, S. (1998). Statistical analysis of the plasmoid evolution with Geotail observations. Journal of Geophysical Research: Space Physics, 103(A3), 44534465. Retrieved from https://agupubs.onlinelibrary.wiley.com/doi/abs/ 10.1029/97JA03240 doi: https://doi.org/10.1029/97JA03240

Imber, S. M., Slavin, J. A., Auster, H. U., \& Angelopoulos, V. (2011).

THEMIS survey of flux ropes and traveling compression regions: Location of the near-Earth reconnection site during solar minimum. Journal of Geophysical Research: Space Physics, 116(A2). Retrieved from https:// agupubs.onlinelibrary.wiley.com/doi/abs/10.1029/2010JA016026 doi: 
https://doi.org/10.1029/2010JA016026

Jabbar, H., \& Khan, R. Z. (2015). Methods to avoid over-fitting and under-fitting in supervised machine learning (comparative study). Computer Science, Communication and Instrumentation Devices, 163-172.

Jackman, C. M., Achilleos, N., Cowley, S. W., Bunce, E. J., Radioti, A., Grodent, D., .. Pryor, W. (2013). Auroral counterpart of magnetic field dipolarizations in Saturn's tail. Planetary and Space Science, 82-83, 34 - 42. Retrieved from http://www.sciencedirect.com/science/article/pii/S003206331300069X doi: https://doi.org/10.1016/j.pss.2013.03.010

Jackman, C. M., \& Arridge, C. S. (2011b). Statistical properties of the magnetic field in the Kronian magnetotail lobes and current sheet. Journal of Geophysical Research: Space Physics, 116(A5). Retrieved from https:// agupubs.onlinelibrary.wiley.com/doi/abs/10.1029/2010JA015973 doi: https://doi.org/10.1029/2010JA015973

Jackman, C. M., Arridge, C. S., Krupp, N., Bunce, E. J., Mitchell, D. G., McAndrews, H. J., ... Coates, A. J. (2008). A multi-instrument view of tail reconnection at Saturn. Journal of Geophysical Research (Space Physics), 113(A11), A11213. doi: 10.1029/2008JA013592

Jackman, C. M., Lamy, L., Freeman, M. P., Zarka, P., Cecconi, B., Kurth, W. S., ... Dougherty, M. K. (2009). On the character and distribution of lowerfrequency radio emissions at Saturn and their relationship to substorm-like events. Journal of Geophysical Research: Space Physics, 114(A8). Retrieved from https://agupubs.onlinelibrary.wiley.com/doi/abs/10.1029/ 2008JA013997 doi: https://doi.org/10.1029/2008JA013997

Jackman, C. M., Russell, C. T., Southwood, D. J., Arridge, C. S., Achilleos, N., \& Dougherty, M. K. (2007). Strong rapid dipolarizations in Saturn's magnetotail: In situ evidence of reconnection. Geophysical Research Letters, $34(11)$. Retrieved from https://agupubs.onlinelibrary.wiley.com/doi/abs/ 10.1029/2007GL029764 doi: 10.1029/2007GL029764

Jackman, C. M., Slavin, J. A., \& Cowley, S. W. H. (2011a). Cassini observations of plasmoid structure and dynamics: Implications for the role of magnetic reconnection in magnetospheric circulation at Saturn. Journal of Geophysical Research: Space Physics, 116(A10). Retrieved from https:// 
agupubs.onlinelibrary.wiley.com/doi/abs/10.1029/2011JA016682 doi: https://doi.org/10.1029/2011JA016682

Jackman, C. M., Slavin, J. A., Kivelson, M. G., Southwood, D. J., Achilleos, N., Thomsen, M. F., .. Vogt, M. F. (2014). Saturn's dynamic magnetotail: A comprehensive magnetic field and plasma survey of plasmoids and traveling compression regions and their role in global magnetospheric dynamics. Journal of Geophysical Research: Space Physics, 119(7), 5465-5494. Retrieved from https://agupubs.onlinelibrary.wiley.com/doi/abs/10.1002/ 2013JA019388 doi: https://doi.org/10.1002/2013JA019388

Jackman, C. M., Thomsen, M. F., \& Dougherty, M. K. (2019). Survey of Saturn's magnetopause and bow shock positions over the entire Cassini mission: Boundary statistical properties and exploration of associated upstream conditions. Journal of Geophysical Research: Space Physics, 124(11), 8865-8883. Retrieved from https://agupubs.onlinelibrary.wiley.com/doi/abs/ 10.1029/2019JA026628 doi: https://doi.org/10.1029/2019JA026628

Jackman, C. M., Thomsen, M. F., Mitchell, D. G., Sergis, N., Arridge, C. S., Felici, M., .. Dougherty, M. K. (2015). Field dipolarization in Saturn's magnetotail with planetward ion flows and energetic particle flow bursts: Evidence of quasi-steady reconnection. Journal of Geophysical Research: Space Physics, 120(5), 3603-3617. Retrieved from https:// agupubs.onlinelibrary.wiley.com/doi/abs/10.1002/2015JA020995 doi: 10.1002/2015JA020995

Jasinski, J. M., Akhavan-Tafti, M., Sun, W., Slavin, J. A., Coates, A. J., Fuselier, S. A., .. Murphy, N. (2021). Flux transfer events at a reconnectionsuppressed magnetopause: Cassini observations at Saturn. Journal of Geophysical Research: Space Physics, 126(2), e2020JA028786. Retrieved from https://agupubs.onlinelibrary.wiley.com/doi/abs/10.1029/ 2020JA028786 (e2020JA028786 2020JA028786) doi: https://doi.org/10.1029/ 2020JA028786

Jasinski, J. M., Arridge, C. S., Bader, A., Smith, A. W., Felici, M., Kinrade, J., ... Murphy, N. (2019). Saturn's open-closed field line boundary: A Cassini electron survey at Saturn's magnetosphere. Journal of Geophysical Research: Space Physics, 124(12), 10018-10035.

Retrieved from https:// 
agupubs.onlinelibrary.wiley.com/doi/abs/10.1029/2019JA027090 doi: https://doi.org/10.1029/2019JA027090

Jasinski, J. M., Slavin, J. A., Arridge, C. S., Poh, G., Jia, X., Sergis, N., ...

Waite Jr., J. H. (2016). Flux transfer event observation at Saturn's dayside magnetopause by the Cassini spacecraft. Geophysical Research Letters, 43(13), 6713-6723. Retrieved from https://agupubs.onlinelibrary .wiley.com/doi/abs/10.1002/2016GL069260 doi: https://doi.org/10.1002/ 2016GL069260

Jia, X., Hansen, K. C., Gombosi, T. I., Kivelson, M. G., Tóth, G., DeZeeuw, D. L., \& Ridley, A. J. (2012). Magnetospheric configuration and dynamics of Saturn's magnetosphere: A global MHD simulation. Journal of Geophysical Research: Space Physics, 117(A5). Retrieved from https:// agupubs.onlinelibrary.wiley.com/doi/abs/10.1029/2012JA017575 doi: https://doi.org/10.1029/2012JA017575

Johnson, J. R., Wing, S., \& Delamere, P. A. $\quad$ (2014, 01). Kelvin Helmholtz instability in planetary magnetospheres. Space Science Reviews, 184(1), 1-31. Retrieved from https://doi.org/10.1007/s11214-014-0085-z doi: 10.1007/ s11214-014-0085-Z

Jurac, S., \& Richardson, J. D. (2005). $\quad$ A self-consistent model of plasma and neutrals at Saturn: Neutral cloud morphology. Journal of Geophysical Research: Space Physics, 110(A9). Retrieved from https:// agupubs.onlinelibrary.wiley.com/doi/abs/10.1029/2004JA010635 doi: https://doi.org/10.1029/2004JA010635

Kaminker, V., Delamere, P. A., Ng, C. S., Dennis, T., Otto, A., \& Ma, X. (2017). Local time dependence of turbulent magnetic fields in saturn's magnetodisc. Journal of Geophysical Research: Space Physics, 122(4), 3972-3984. Retrieved from https://agupubs.onlinelibrary.wiley.com/doi/abs/10.1002/ 2016JA023834 doi: https://doi.org/10.1002/2016JA023834

Kellett, S., Bunce, E. J., Coates, A. J., \& Cowley, S. W. H. (2009). Thickness of Saturn's ring current determined from north-south Cassini passes through the current layer. Journal of Geophysical Research: Space Physics, 114(A4). Retrieved from https://agupubs.onlinelibrary.wiley.com/doi/abs/ 10.1029/2008JA013942 doi: https://doi.org/10.1029/2008JA013942 
Lapedes, A., \& Farber, R. (1987). How neural nets work. In (p. 442-456). doi: 10 .1142/9789814434102_0012

Ma, X., Delamere, P. A., Thomsen, M. F., Otto, A., Neupane, B., Burkholder, B., \& Nykyri, K. (2019). Flux tube entropy and specific entropy in saturn's magnetosphere. Journal of Geophysical Research: Space Physics, 124(3), 15931611. Retrieved from https://agupubs.onlinelibrary.wiley.com/doi/abs/ 10.1029/2018JA026150 doi: https://doi.org/10.1029/2018JA026150

Masters, A. (2018). A more viscous-like solar wind interaction with all the giant planets. Geophysical Research Letters, 45(15), 7320-7329. Retrieved from https://agupubs.onlinelibrary.wiley.com/doi/abs/10.1029/ 2018GL078416 doi: https://doi.org/10.1029/2018GL078416

Masters, A., Mitchell, D. G., Coates, A. J., \& Dougherty, M. K. (2011). Saturn's low-latitude boundary layer: 1. properties and variability. Journal of Geophysical Research: Space Physics, 116(A6). Retrieved from https:// agupubs.onlinelibrary.wiley.com/doi/abs/10.1029/2010JA016421 doi: https://doi.org/10.1029/2010JA016421

McAndrews, H., Thomsen, M., Arridge, C., Jackman, C., Wilson, R., Henderson, M., ... Coates, M., A.J. and. Dougherty (2009). Plasma in Saturn's nightside magnetosphere and the implications for global circulation. Planetary and Space Science, 57(14), 1714-1722. Retrieved from https:// wWw.sciencedirect.com/science/article/pii/S0032063309000750 doi: https://doi.org/10.1016/j.pss.2009.03.003

McAndrews, H. J., Owen, C. J., Thomsen, M. F., Lavraud, B., Coates, A. J., Dougherty, M. K., \& Young, D. T. (2008). Evidence for reconnection at Saturn's magnetopause. Journal of Geophysical Research: Space Physics, 113(A4). Retrieved from https://agupubs.onlinelibrary.wiley.com/doi/ abs/10.1029/2007JA012581 doi: https://doi.org/10.1029/2007JA012581

Milan, S. E., Provan, G., \& Hubert, B. (2007). Magnetic flux transport in the Dungey cycle: A survey of dayside and nightside reconnection rates. Journal of Geophysical Research: Space Physics, 112(A1). Retrieved from https:// agupubs.onlinelibrary.wiley.com/doi/abs/10.1029/2006JA011642 doi: 10.1029/2006JA011642

Milovanov, A. V., Zelenyi, L. M., \& Zimbardo, G. $\quad$ (1996). Fractal structures 
and power law spectra in the distant Earth's magnetotail.

Journal of Geo-

physical Research: Space Physics, 101(A9), 19903-19910.

Retrieved from

https://agupubs.onlinelibrary.wiley.com/doi/abs/10.1029/96JA01562

doi: https://doi.org/10.1029/96JA01562

Neupane, B. R., Delamere, P. A., Ma, X., Ng, C.-S., Burkholder, B., \& Damiano, P. (2021). On the nature of turbulent heating and radial transport in saturn's magnetosphere. Journal of Geophysical Research: Space Physics, 126(1), e2020JA027986. Retrieved from https://agupubs.onlinelibrary.wiley .com/doi/abs/10.1029/2020JA027986 (e2020JA027986 2020JA027986) doi: https://doi.org/10.1029/2020JA027986

Neupane, B. R., Delamere, P. A., Wilson, R. J., \& Ma, X. (2019). Quantifying mass and magnetic flux transport in Saturn's magnetosphere. Journal of Geophysical Research: Space Physics, 124(3), 1916-1926. Retrieved from https:// agupubs.onlinelibrary.wiley.com/doi/abs/10.1029/2018JA026022 doi: https://doi.org/10.1029/2018JA026022

Pontius Jr., D. H., \& Hill, T. W. (2009). Plasma mass loading from the extended neutral gas torus of Enceladus as inferred from the observed plasma corotation lag. Geophysical Research Letters, 36(23). Retrieved from https:// agupubs.onlinelibrary.wiley.com/doi/abs/10.1029/2009GL041030 doi: https://doi.org/10.1029/2009GL041030

Provan, G., Cowley, S. W. H., Bradley, T. J., Bunce, E. J., Hunt, G. J., \& Dougherty, M. K. (2018). Planetary period oscillations in Saturn's magnetosphere: Cassini magnetic field observations over the northern summer solstice interval. Journal of Geophysical Research: Space Physics, 123(5), 3859-3899. Retrieved from https://agupubs.onlinelibrary.wiley.com/doi/abs/ 10.1029/2018JA025237 doi: https://doi.org/10.1029/2018JA025237

Reed, J. J., Jackman, C. M., Lamy, L., Kurth, W. S., \& Whiter, D. K. (2018). Lowfrequency extensions of the Saturn kilometric radiation as a proxy for magnetospheric dynamics. Journal of Geophysical Research: Space Physics, 123(1), 443-463. Retrieved from https://agupubs.onlinelibrary.wiley.com/doi/ abs/10.1002/2017JA024499 doi: https://doi.org/10.1002/2017JA024499

Richardson, I. G., Cowley, S. W. H., Hones Jr., E. W., \& Bame, S. J.

(1987).

Plasmoid-associated energetic ion bursts in the deep geomagnetic tail: Proper- 
ties of plasmoids and the postplasmoid plasma sheet. Journal of Geophysical Research: Space Physics, 92(A9), 9997-10013. Retrieved from https:// agupubs. onlinelibrary.wiley.com/doi/abs/10.1029/JA092iA09p09997 doi: https://doi.org/10.1029/JA092iA09p09997

Russell, C. T., Jackman, C. M., Wei, H. Y., Bertucci, C., \& Dougherty, M. K. (2008). Titan's influence on Saturnian substorm occurrence. Geophysical Research Letters, 35(12). Retrieved from https://agupubs.onlinelibrary .wiley.com/doi/abs/10.1029/2008GL034080 doi: 10.1029/2008GL034080

Schneider, S., Greenberg, S., Taylor, G. W., \& Kremer, S. C. $\quad$ (2020). Three critical factors affecting automated image species recognition performance for camera traps. Ecology and Evolution, 10(7), 3503-3517. Retrieved from https://onlinelibrary.wiley.com/doi/abs/10.1002/ece3.6147 doi: https://doi.org/10.1002/ece3.6147

Sergis, N., Arridge, C. S., Krimigis, S. M., Mitchell, D. G., Rymer, A. M., Hamilton, D. C., ... Coates, A. J. (2011). Dynamics and seasonal variations in Saturn's magnetospheric plasma sheet, as measured by Cassini. Journal of Geophysical Research: Space Physics, 116(A4). Retrieved from https:// agupubs.onlinelibrary.wiley.com/doi/abs/10.1029/2010JA016180 doi: https://doi.org/10.1029/2010JA016180

Slavin, J. A., Smith, E. J., Tsurutani, B. T., Sibeck, D. G., Singer, H. J., Baker, D. N., .. Scarf, F. L. (1984). Substorm associated traveling compression regions in the distant tail: Isee-3 Geotail observations. Geophysical Research Letters, 11(7), 657-660. Retrieved from https://agupubs.onlinelibrary.wiley .com/doi/abs/10.1029/GL011i007p00657 doi: 10.1029/GL011i007p00657

Smith, A. W., Jackman, C. M., Frohmaier, C. M., Fear, R. C., Slavin, J. A., \& Coxon, J. C. (2018c). Evaluating single spacecraft observations of planetary magnetotails with simple Monte Carlo simulations: 2. magnetic flux rope signature selection effects. Journal of Geophysical Research: Space Physics, 123(12), 10,124-10,138. Retrieved from https:// agupubs.onlinelibrary.wiley.com/doi/abs/10.1029/2018JA025959 doi: https://doi.org/10.1029/2018JA025959

Smith, A. W., Jackman, C. M., \& Thomsen, M. F. (2016). Magnetic reconnection in Saturn's magnetotail: A comprehensive magnetic field survey. Journal of Geo- 
physical Research: Space Physics, 121(4), 2984-3005. Retrieved from https:// agupubs.onlinelibrary.wiley.com/doi/abs/10.1002/2015JA022005 doi: 10.1002/2015JA022005

Smith, A. W., Jackman, C. M., Thomsen, M. F., Lamy, L., \& Sergis, N. (2018a). Multi-instrument investigation of the location of Saturn's magnetotail xline. Journal of Geophysical Research: Space Physics, 123(7), 5494-5505. Retrieved from https://agupubs.onlinelibrary.wiley.com/doi/abs/ 10.1029/2018JA025532 doi: 10.1029/2018JA025532

Smith, A. W., Jackman, C. M., Thomsen, M. F., Sergis, N., Mitchell, D. G., \& Roussos, E. (2018b). Dipolarization fronts with associated energized electrons in Saturn's magnetotail. Journal of Geophysical Research: Space Physics, 123(4), 2714-2735. Retrieved from https://agupubs.onlinelibrary.wiley .com/doi/abs/10.1002/2017JA024904 doi: 10.1002/2017JA024904

Smith, A. W., Slavin, J. A., Jackman, C. M., Fear, R. C., Poh, G.-K., DiBraccio, G. A., .. Trenchi, L. (2017). Automated force-free flux rope identification. Journal of Geophysical Research: Space Physics, 122(1), 780-791. Retrieved from https://agupubs.onlinelibrary.wiley.com/doi/abs/10.1002/ 2016JA022994 doi: https://doi.org/10.1002/2016JA022994

Staniland, N. R., Dougherty, M. K., Masters, A., \& Bunce, E. J. $\quad$ (2020). Determining the nominal thickness and variability of the magnetodisc current sheet at Saturn. Journal of Geophysical Research: Space Physics, 125(5), e2020JA027794. Retrieved from https://agupubs.onlinelibrary .wiley.com/doi/abs/10.1029/2020JA027794 (e2020JA027794 10.1029/2020JA027794) doi: https://doi.org/10.1029/2020JA027794

Szego, K., Nemeth, Z., Erdos, G., Foldy, L., Bebesi, Z., Thomsen, M., \& Delapp, D. (2012). Location of the magnetodisk in the nightside outer magnetosphere of Saturn near equinox based on ion densities. Journal of Geophysical Research: Space Physics, 117(A9). Retrieved from https:// agupubs.onlinelibrary.wiley.com/doi/abs/10.1029/2012JA017817 doi: https://doi.org/10.1029/2012JA017817

Thomsen, M. F., Jackman, C. M., Tokar, R. L., \& Wilson, R. J. (2014). Plasma flows in Saturn's nightside magnetosphere. Journal of Geophysical Research: Space Physics, 119(6), 4521-4535.

Retrieved from https:// 
agupubs.onlinelibrary.wiley.com/doi/abs/10.1002/2014JA019912 doi: https://doi.org/10.1002/2014JA019912

Vasyliunas, V. M. (1983). Plasma distribution and flow. In A. J. Dessler (Ed.), Physics of the Jovian Magnetosphere (p. 395-453). Cambridge University Press. doi: 10.1017/CBO9780511564574.013

Vogt, M. F., Connerney, J. E., DiBraccio, G. A., Wilson, R. J., Thomsen, M. F., Ebert, R. W., .. Bolton, S. J. (2020). Magnetotail reconnection at Jupiter: A survey of Juno magnetic field observations. Journal of Geophysical Research: Space Physics, 125(3), e2019JA027486. Retrieved from https:// agupubs .onlinelibrary .wiley .com/doi/abs/10.1029/2019JA027486 (e2019JA027486 2019JA027486) doi: https://doi.org/10.1029/2019JA027486

Vogt, M. F., Kivelson, M. G., Khurana, K. K., Joy, S. P., \& Walker, R. J. (2010). Reconnection and flows in the Jovian magnetotail as inferred from magnetometer observations. Journal of Geophysical Research: Space Physics, 115(A6). Retrieved from https://agupubs.onlinelibrary.wiley.com/doi/abs/ 10.1029/2009JA015098 doi: https://doi.org/10.1029/2009JA015098

Yao, Z. H., Grodent, D., Ray, L. C., Rae, I. J., Coates, A. J., Pu, Z. Y., .. Dunn, W. R. (2017). Two fundamentally different drivers of dipolarizations at Saturn. Journal of Geophysical Research: Space Physics, 122(4), 4348-4356. Retrieved from https://agupubs.onlinelibrary.wiley.com/doi/abs/ 10.1002/2017JA024060 doi: $10.1002 / 2017 J A 024060$

Ying, X. (2019). An overview of overfitting and its solutions. In Journal of physics: Conference series (Vol. 1168, p. 022022).

Zieger, B., Hansen, K. C., Gombosi, T. I., \& De Zeeuw, D. L. $\quad$ (2010). Periodic plasma escape from the mass-loaded Kronian magnetosphere. Journal of Geophysical Research: Space Physics, 115(A8). Retrieved from https://agupubs .onlinelibrary.wiley.com/doi/abs/10.1029/2009JA014951 doi: https:// doi.org/10.1029/2009JA014951 


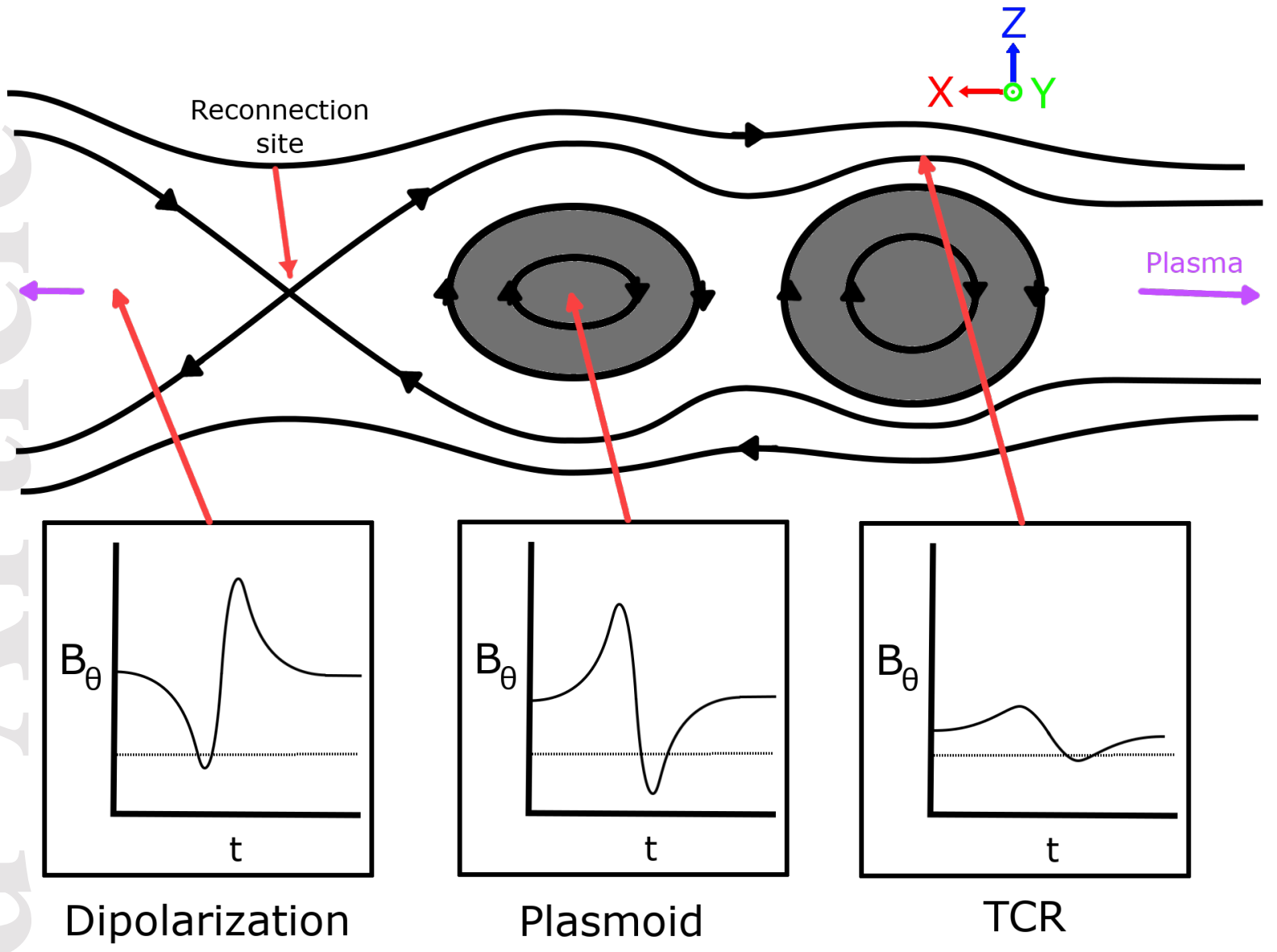

This article is protected by copyright. All rights reserved. 


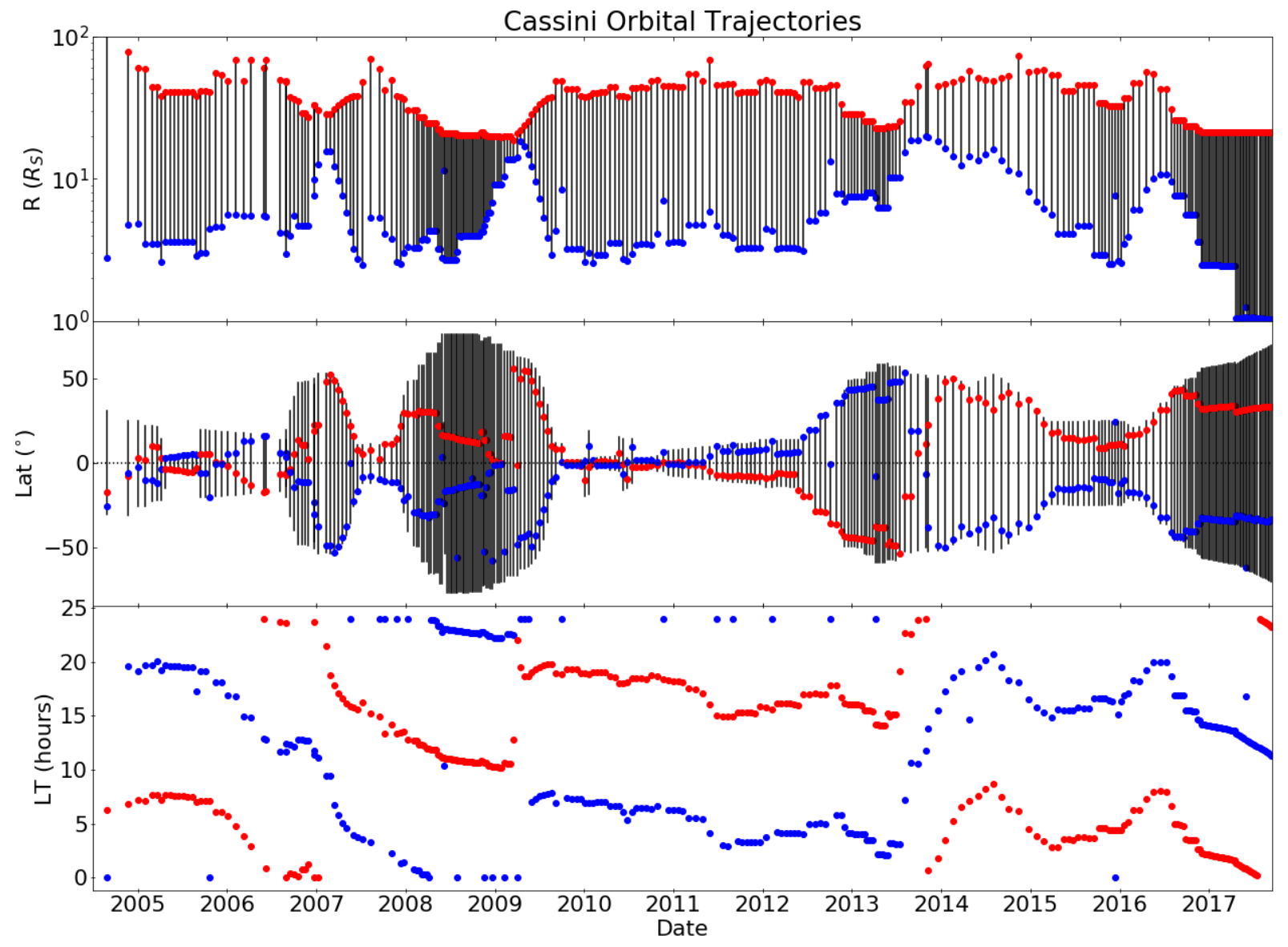

This article is protected by copyright. All rights reserved. 


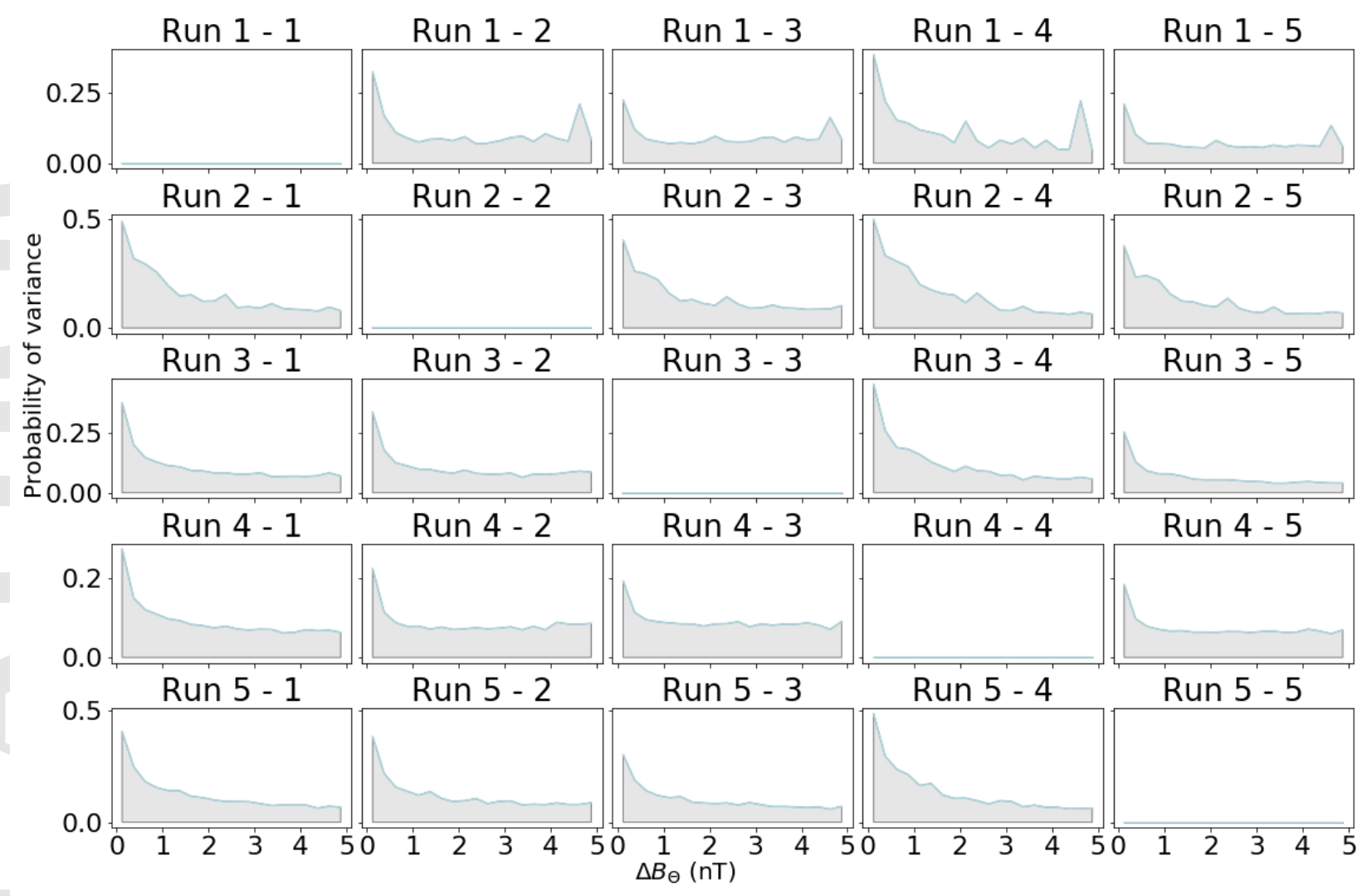

This article is protected by copyright. All rights reserved. 

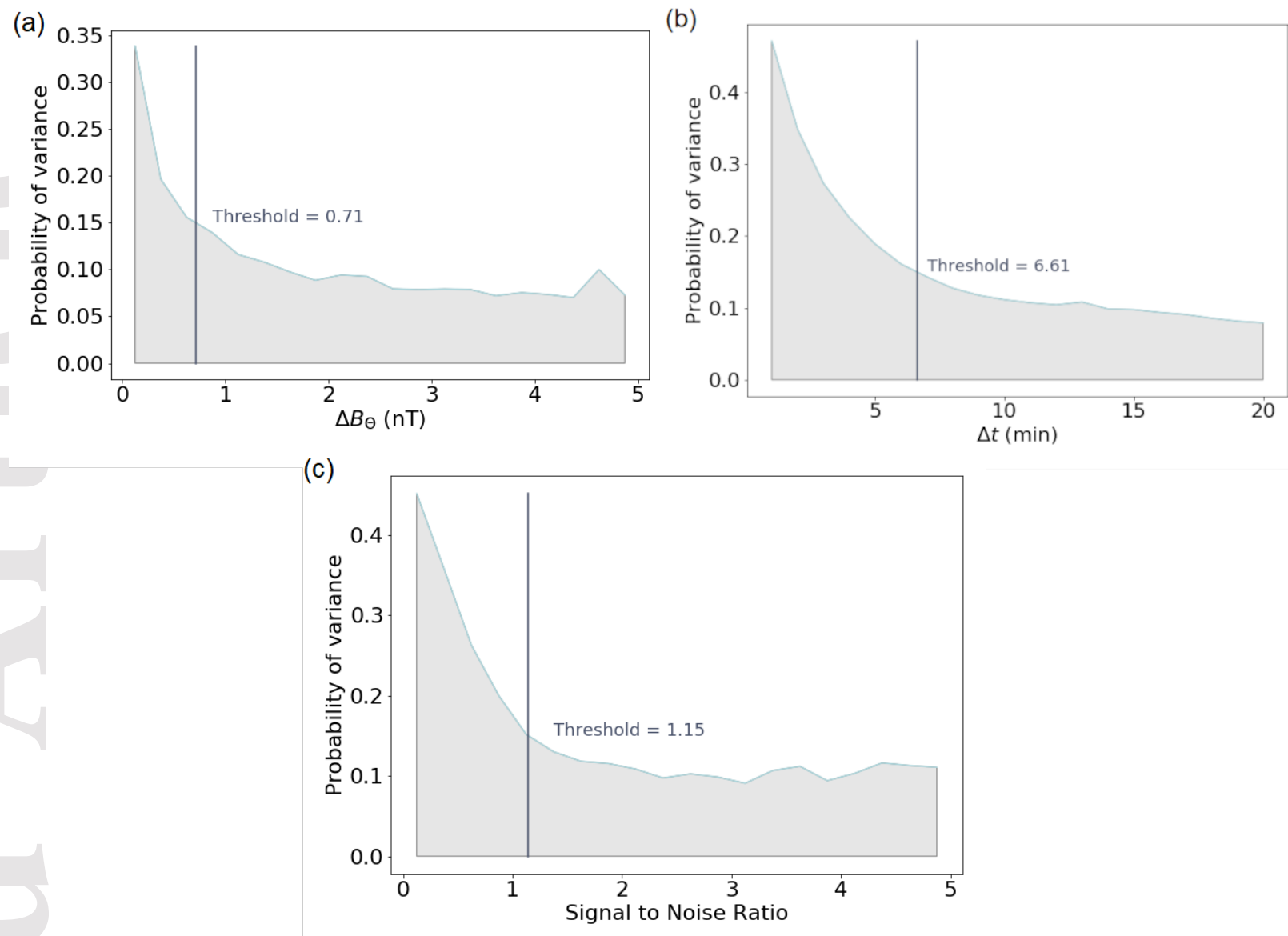

This article is protected by copyright. All rights reserved. 


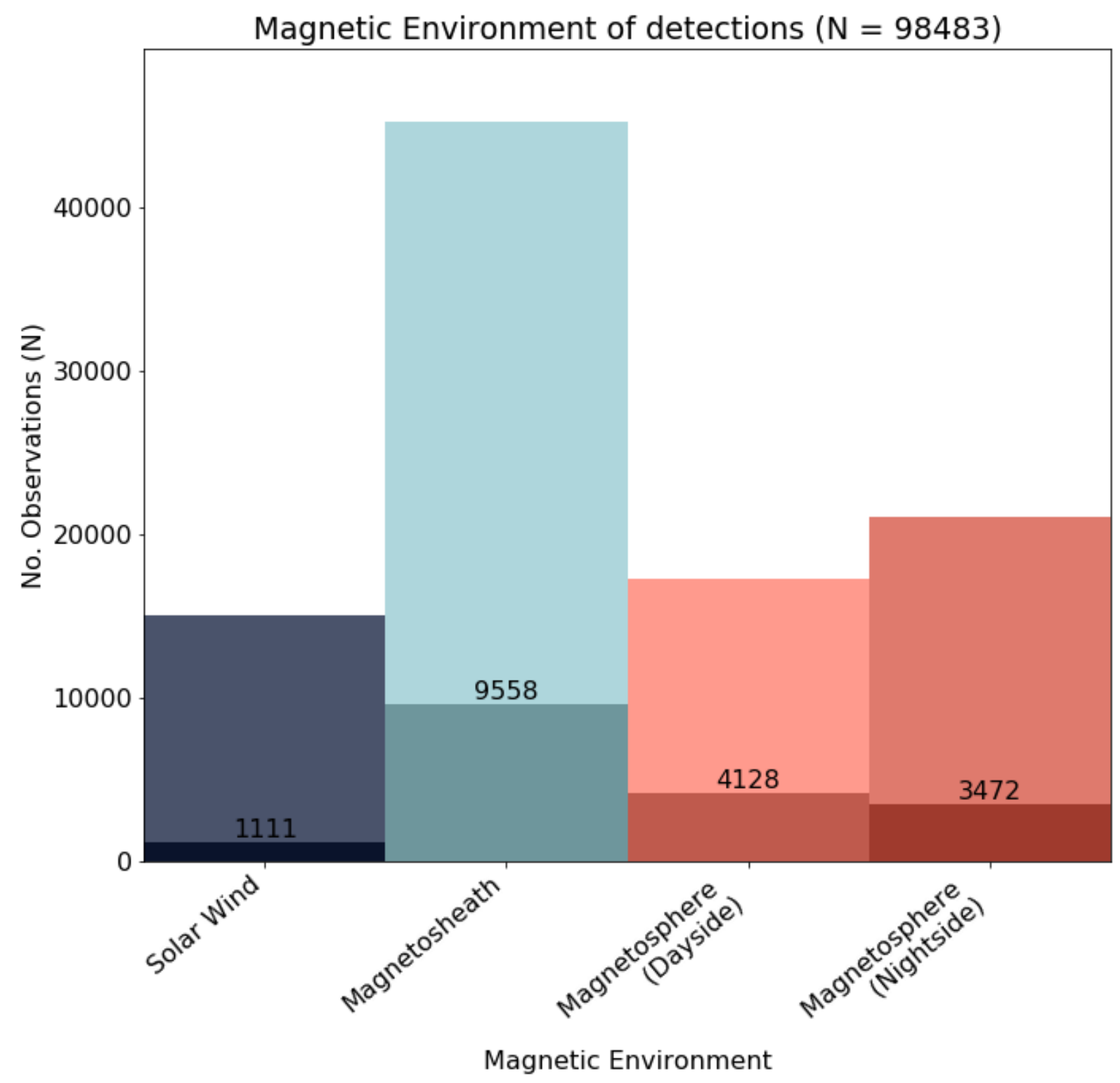

This article is protected by copyright. All rights reserved. 


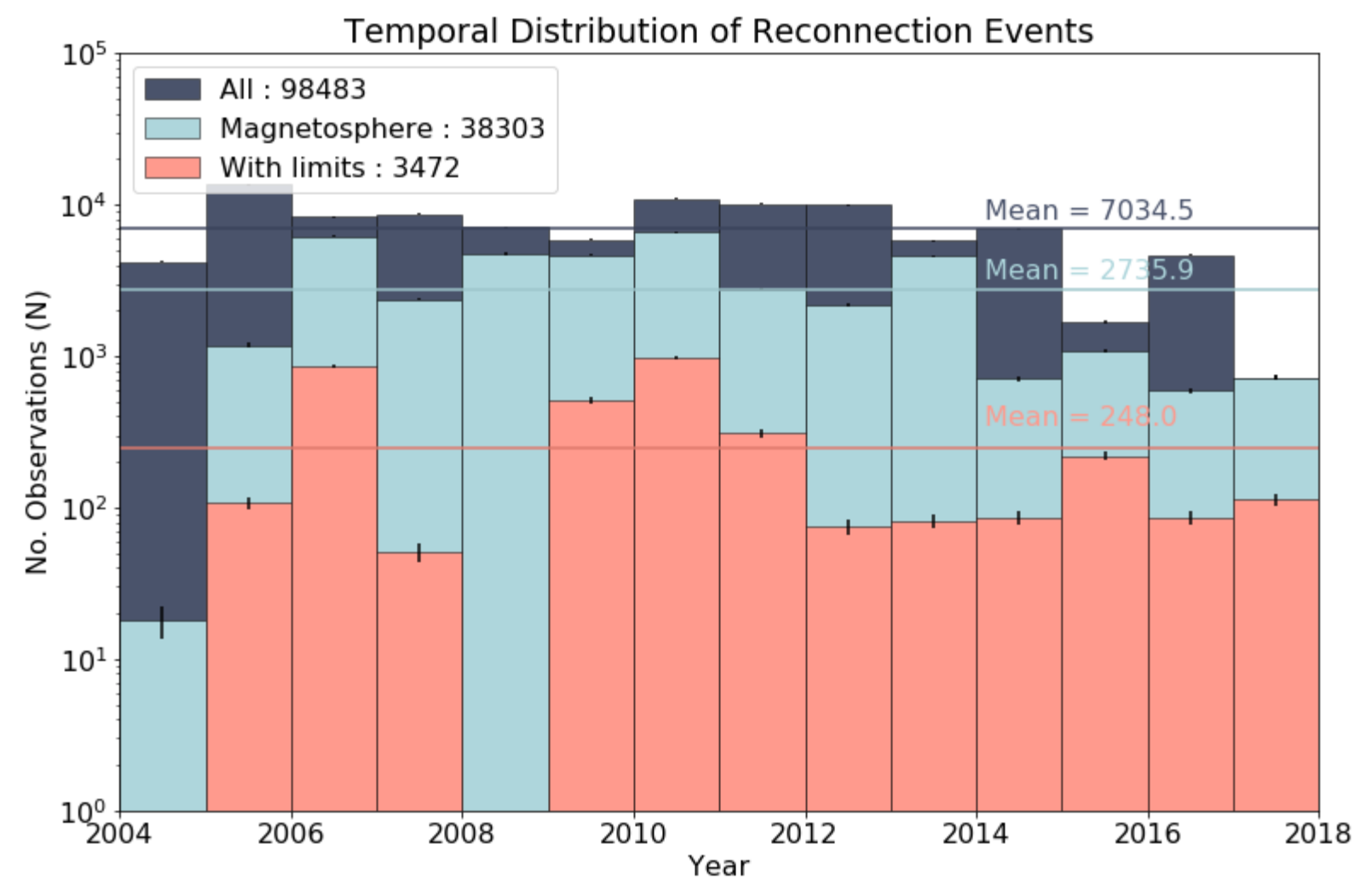

This article is protected by copyright. All rights reserved. 
Latitude of night-side reconnection events within the magnetosphere

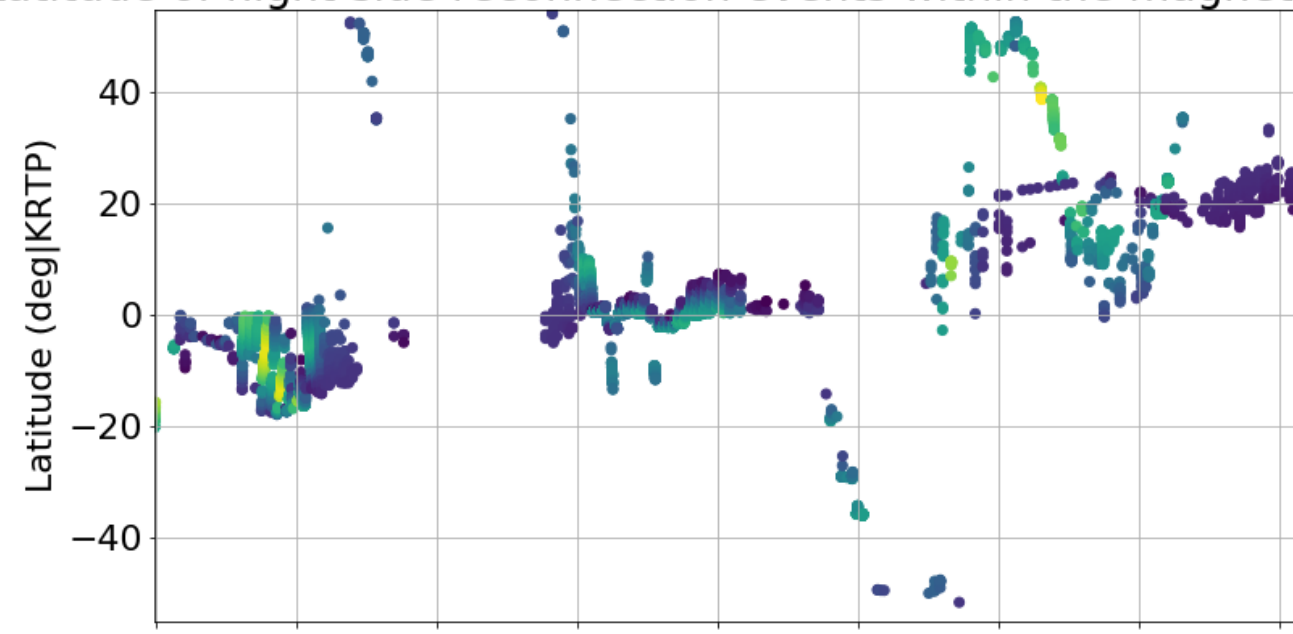

60

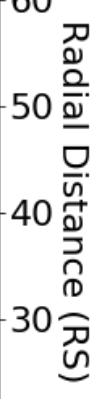

20

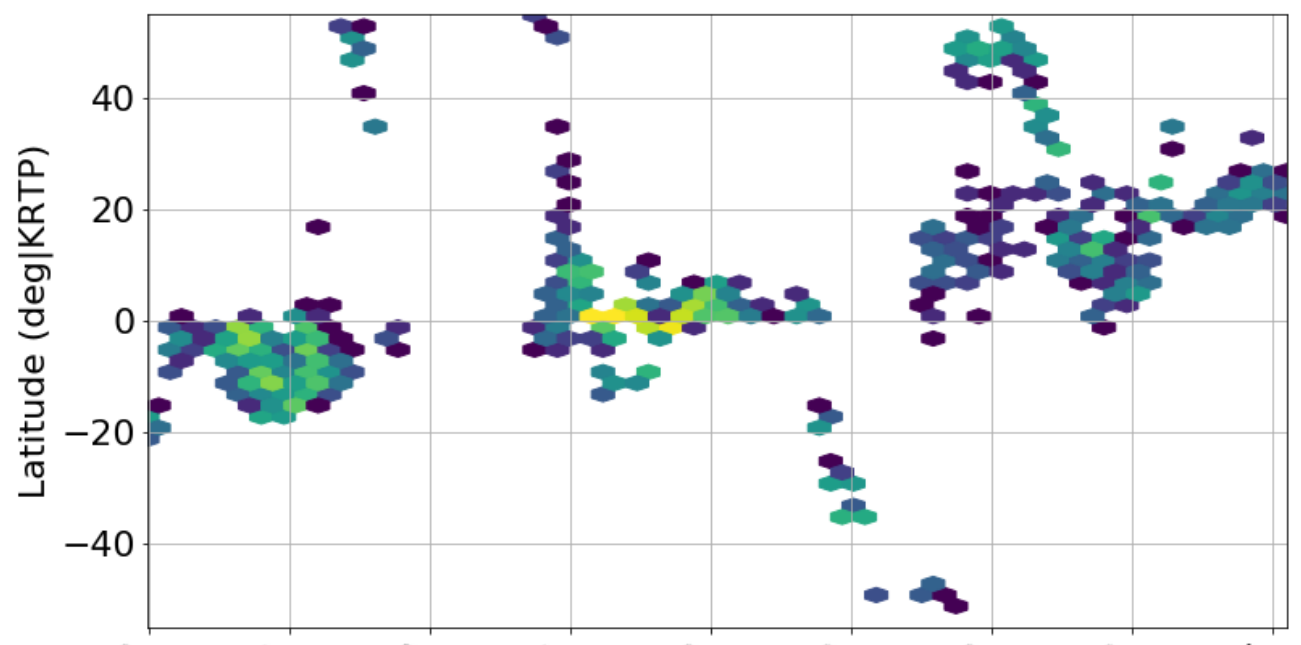

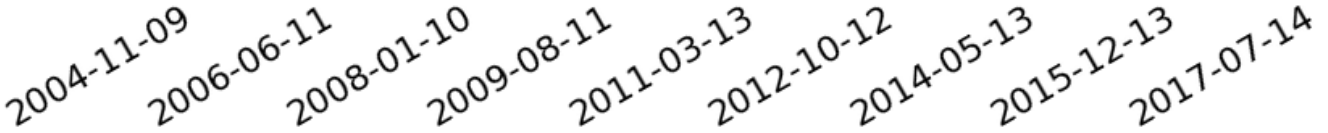

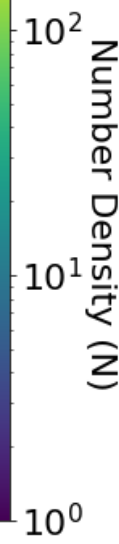

Date

This article is protected by copyright. All rights reserved. 

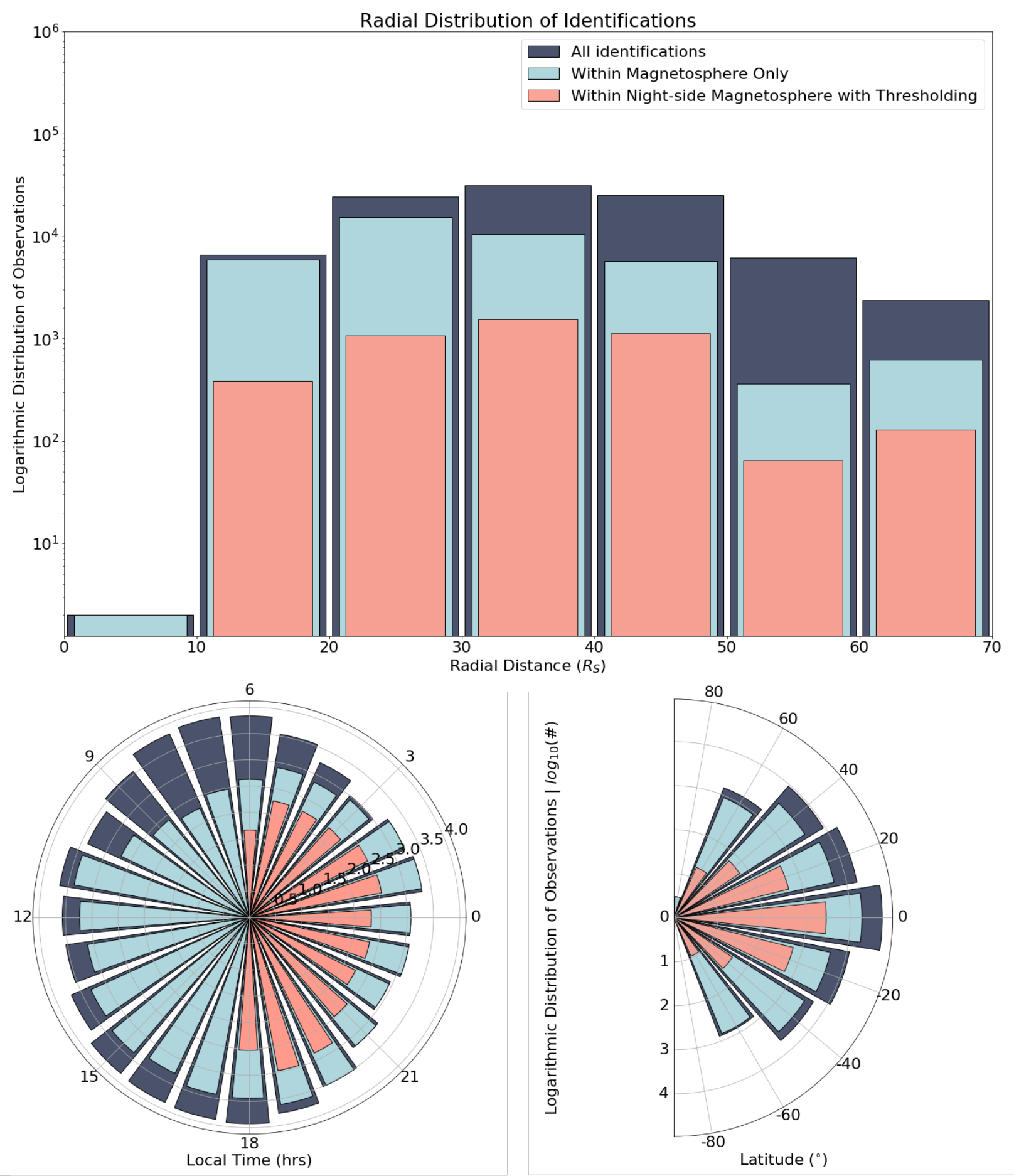

This article is protected by copyright. All rights reserved. 
Normalized Reconnection Occurence Distribution
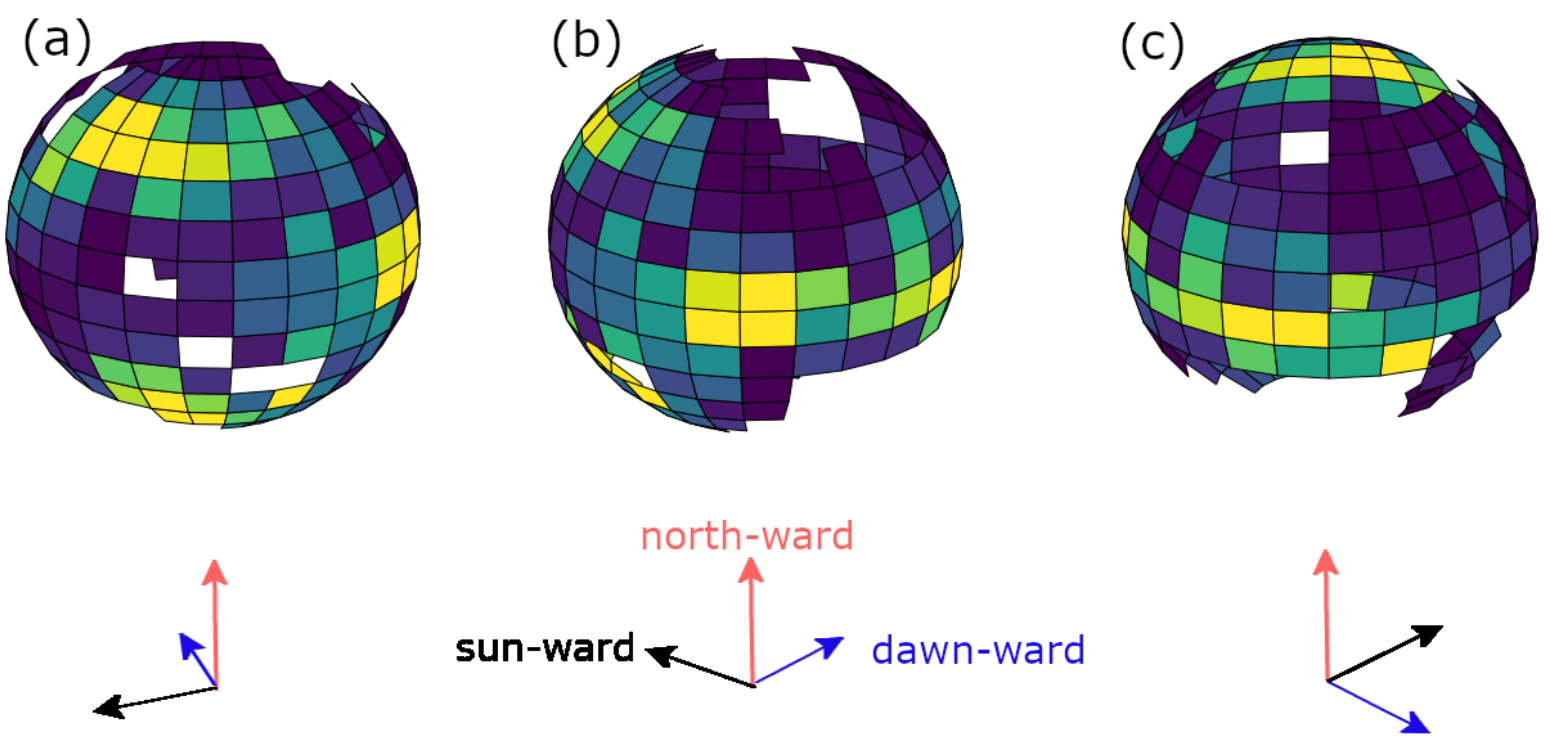

This article is protected by copyright. All rights reserved. 

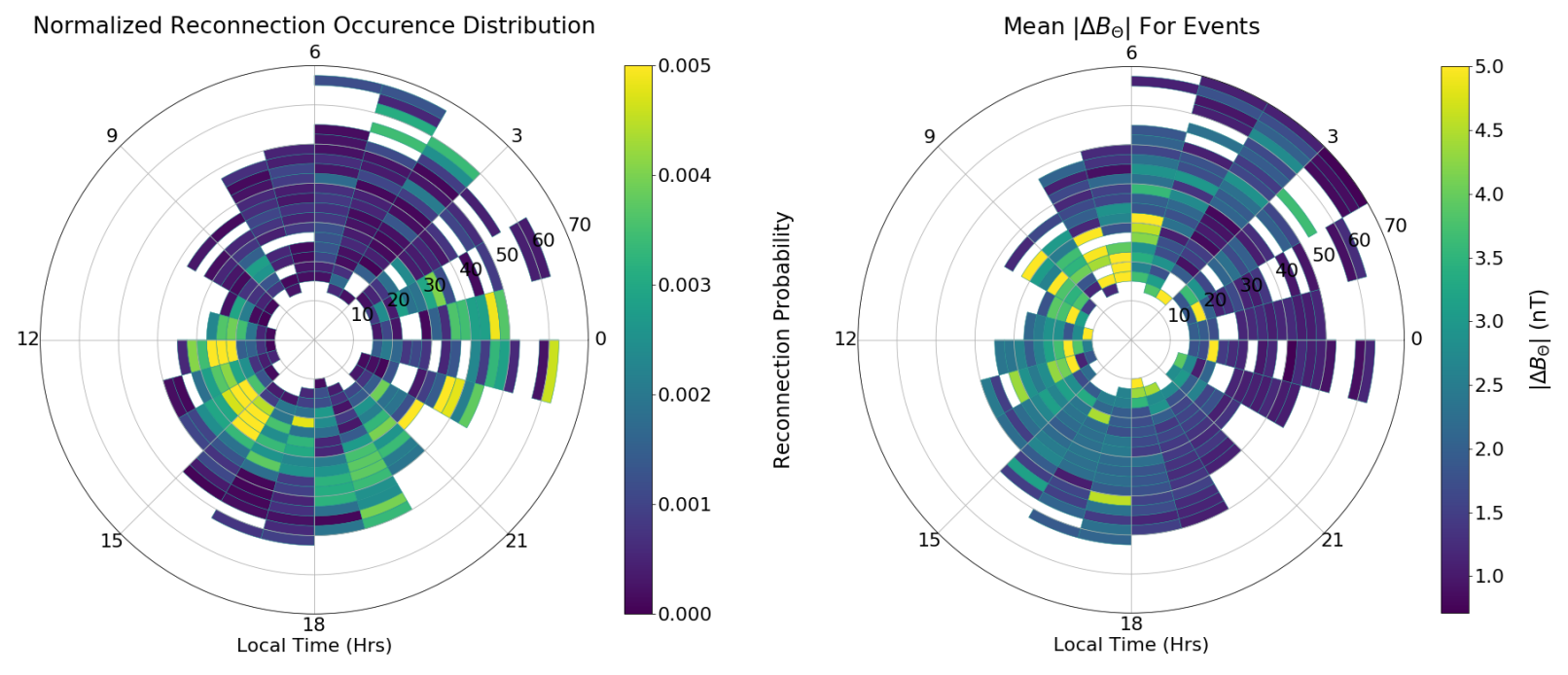

This article is protected by copyright. All rights reserved. 
Normalized Directionality of Magnetotail Events Within Limits (LT: 18 - 6)

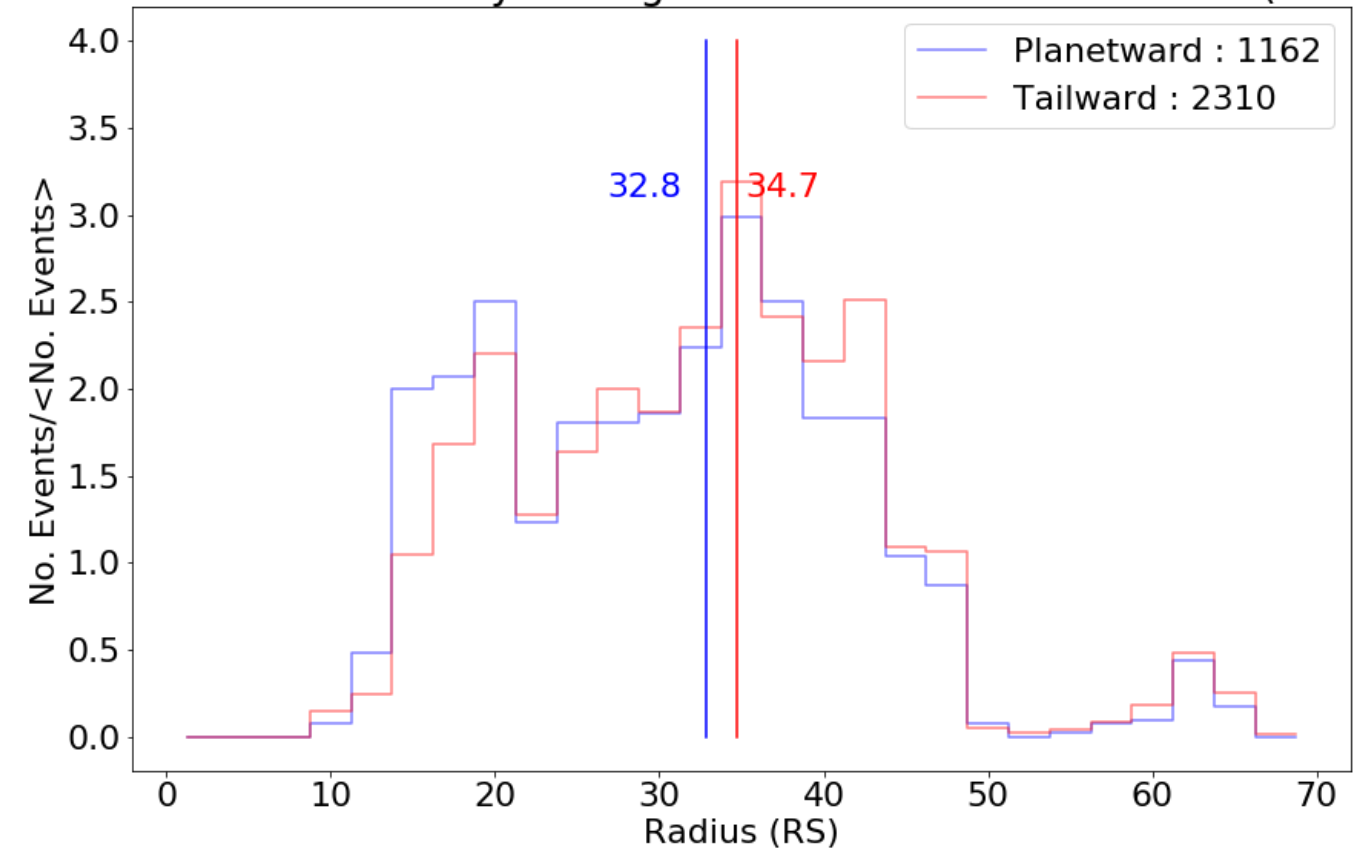

This article is protected by copyright. All rights reserved. 


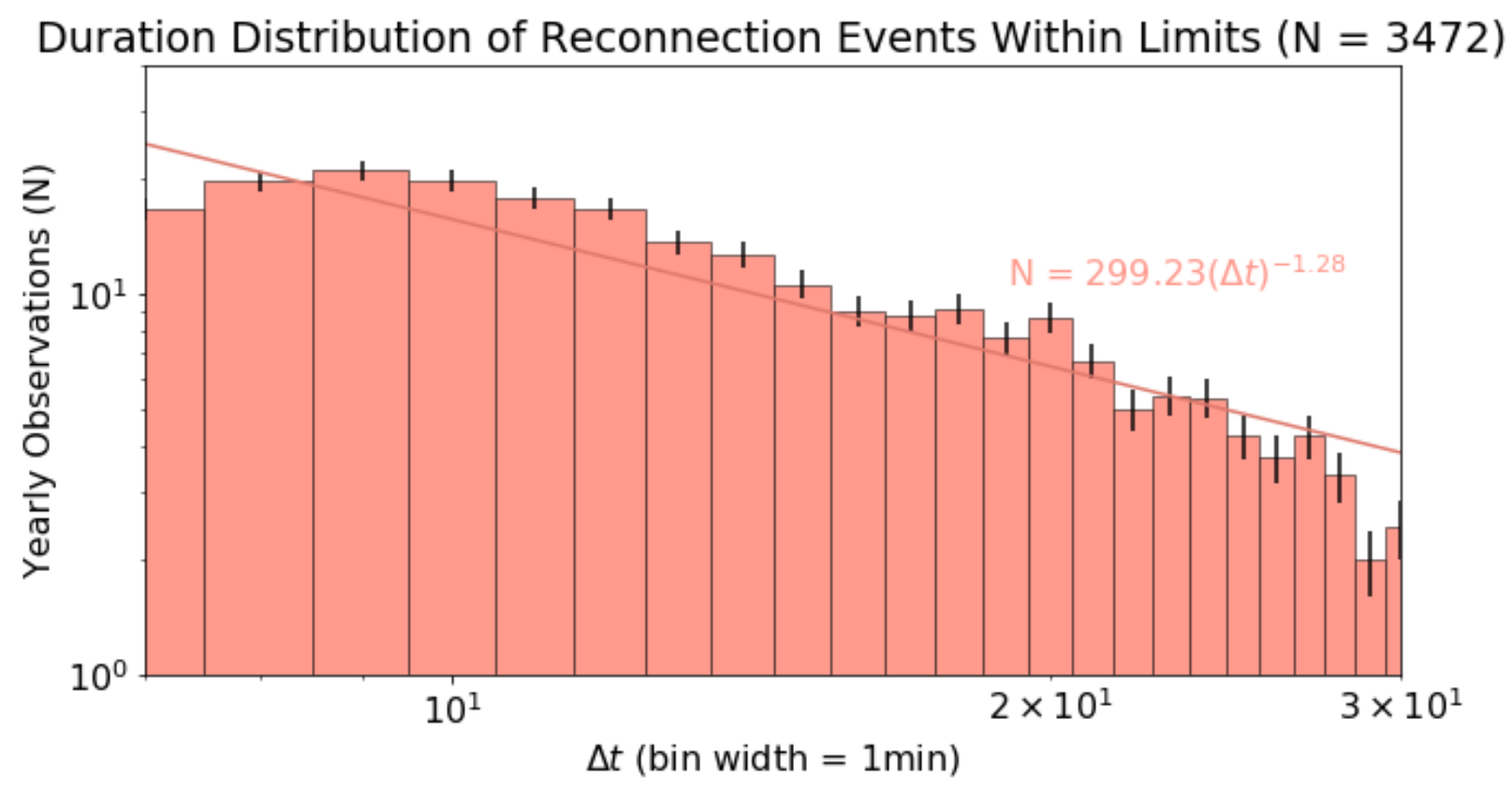

This article is protected by copyright. All rights reserved. 


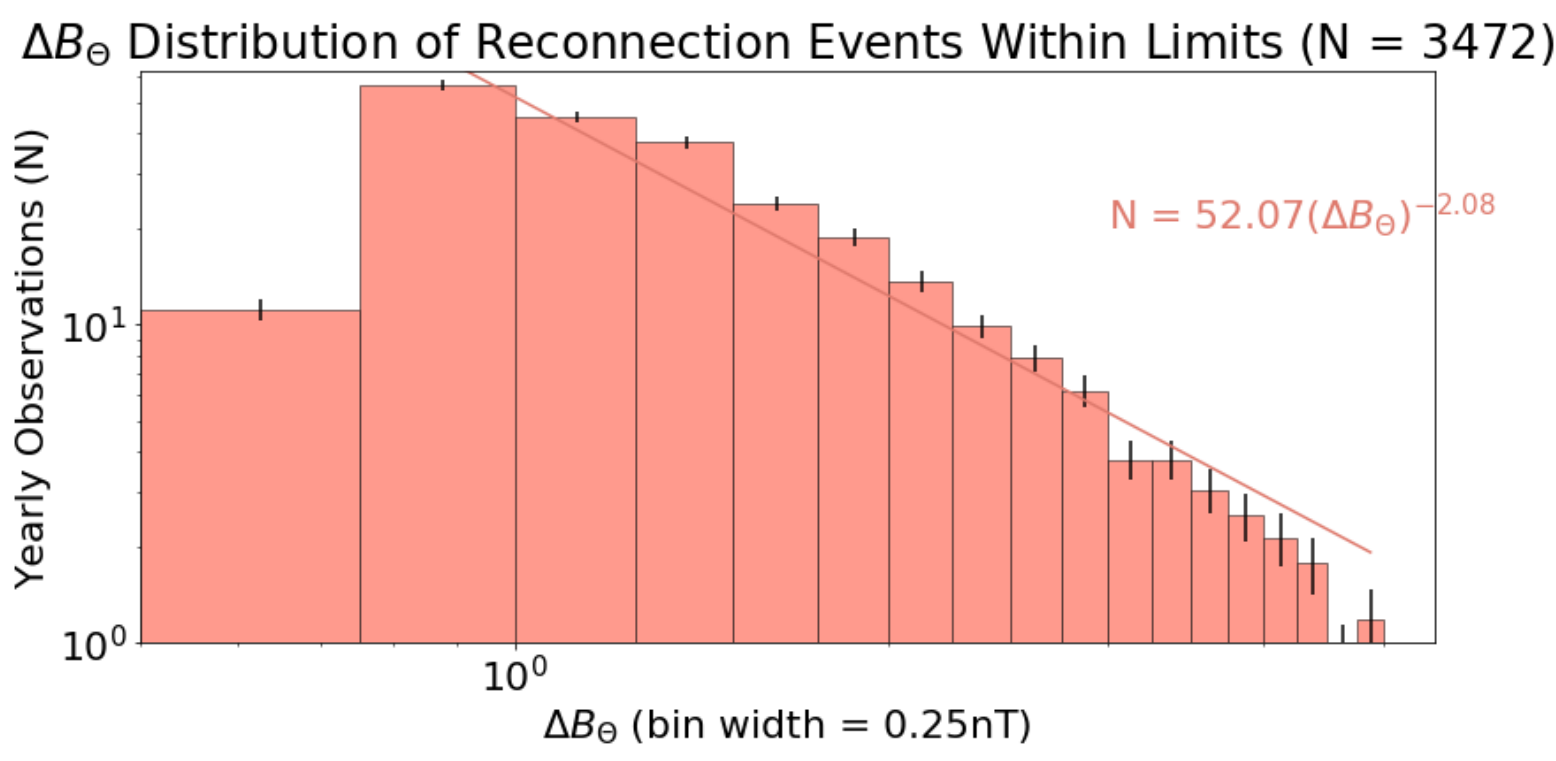

This article is protected by copyright. All rights reserved. 